\title{
EXPECTING THE UNEXPECTED: QUANTIFYING THE PERSISTENCE OF UNEXPECTED HYPERSURFACES
}

\author{
G. FAVACCHIO, E. GUARDO, B. HARBOURNE, AND J. MIGLIORE
}

\begin{abstract}
If $X \subset \mathbb{P}^{n}$ is a reduced subscheme, we say that $X$ admits an unexpected hypersurface of degree $t$ for multiplicity $m$ if the imposition of having multiplicity $m$ at a general point $P$ fails to impose the expected number of conditions on the linear system of hypersurfaces of degree $t$ containing $X$. Conditions which either guarantee the occurrence of unexpected hypersurfaces, or which ensure that they cannot occur, are not well understand. We introduce new methods for studying unexpectedness, such as the use of generic initial ideals and partial elimination ideals to clarify when it can and when it cannot occur. We also exhibit algebraic and geometric properties of $X$ which in some cases guarantee and in other cases preclude $X$ having certain kinds of unexpectedness. In addition, we formulate a new way of quantifying unexpectedness (our AV sequence), which allows us detect the extent to which unexpectedness persists as $t$ increases but $t-m$ remains constant. Finally, we study to what extent we can detect unexpectedness from the Hilbert function of X.
\end{abstract}

\section{INTRODUCTION}

A classical kind of problem in algebraic geometry is to consider vanishing conditions on a linear system $\mathcal{L}$, and to ask if the dimension of the resulting linear system is what one would expect based on the dimension of $\mathcal{L}$ and the specific conditions imposed. That is, one asks if the desired vanishing imposes the expected number of conditions on $\mathcal{L}$. For example, if $\mathcal{L}$ is the complete linear system of conics in $\mathbb{P}^{2}$ (which is 5-dimensional) and $P$ is a point, then vanishing to multiplicity 2 at $P$ imposes three conditions on $\mathcal{L}$; that is, there is a 2-dimensional linear system of conics double at $P$ as expected. However, if we impose vanishing to multiplicity 2 at each of two points $P_{1}$ and $P_{2}$, we expect $3+3=6$ conditions, i.e. we expect there to be no such conic, while in fact the double line passing through $P_{1}$ and $P_{2}$ is such a conic. Continuing in this direction leads to the well-known Segre-Harbourne-Gimigliano-Hirschowitz (SHGH) Conjecture [Se, H, Gi, Hi]. A conjecture of Laface and Ugaglia addresses the corresponding situation in $\mathbb{P}^{3}[\mathrm{LU}]$, while results of Alexander and Hirschowitz [AH] partially address the situation in $\mathbb{P}^{n}$ for all $n \geq 2$, but much remains unknown.

Date: edited: January 28, 2020; compiled January 29, 2020.

2010 Mathematics Subject Classification. Primary: 14C20, 13D40, 14Q10, 14M10; Secondary: 14M05, 14M07, 13E10.

Key words and phrases. unexpected hypersurface, unexpected curve, Hilbert function, generic initial ideal, partial elimination ideal, complete intersection, $\mathrm{AV}$-sequence, SHGH, $O$-sequences, SI-sequences, artinian reductions, degenerate varieties, base conditions, cones.

Acknowledgements: Favacchio and Guardo were partially supported by the Università degli Studi di Catania, "Piano della Ricerca 2016/2018 Linea di intervento 2" and by the "National Group for Algebraic and Geometric Structures, and their Applications" (GNSAGA of INdAM). Harbourne was partially supported by Simons Foundation grant \#524858. Migliore was partially supported by Simons Foundation grant \#309556. 
It is not only vanishing conditions imposed by points that is of interest. Given a general set of $r$ lines in $\mathbb{P}^{n}$, one could ask if vanishing on all of these lines with multiplicity 1 imposes the expected number of conditions on the complete linear system of hypersurfaces of given degree $d$, and an affirmative answer was given by Hartshorne and Hirschowitz [HaHi]. Their paper led to much other work, such as research in which lines of higher multiplicity are allowed (for just two recent examples, see [DHRST] and [BDSSS]). The work of Hartshorne and Hirschowitz also led to the paper [CCG], which can be viewed as a direct precursor to the study of unexpected hypersurfaces.

Thus in recent years, a flurry of activity has emerged on this kind of problem. Some of it grew out of a striking example in [DIV], later formalized in [CHMN] and since then branching off in many different directions (for some examples, see [CM, DMO, DHRST, FGST, HaH, HMNT, HMT, S1, S2, Tr]). The path that led to this paper began by our looking for conditions on a variety $X$ that either automatically force the existence of unexpected hypersurfaces, or else force the conclusion that no unexpected hypersurfaces exist. The results we describe below give examples of such conditions. But more specifically we can ask: are there conditions on the Hilbert function that force either of these outcomes? It turns out that if the Hilbert function forces $X$ to be degenerate in $\mathbb{P}^{n}$ then $X$ does not admit any unexpected hypersurfaces of any kind in $\mathbb{P}^{n}$ (see Corollary 3.5), regardless of whether it does in the smallest linear space containing it. Beyond that, additional conditions involving the geometry of $X$ seem to be involved. Indeed, with a minor assumption on the geometry of $X$, there are such Hilbert functions (see Theorem 7.8), but in the setting of non-degenerate, finite sets of points, we conjecture that there are no Hilbert functions that force any kind of unexpectedness (see Conjecture 7.1).

We now describe our results in more detail. Given a subscheme $X$ of $\mathbb{P}_{K}^{n}$, its defining saturated homogeneous ideal $I_{X} \subseteq R=K\left[\mathbb{P}_{K}^{n}\right]=K\left[x_{0}, \ldots, x_{n}\right]$ (where $K$ is a field) and integers $t \geq m \geq 1$, we define three numbers associated to $(X, t, m)$ (see Notation 2.4). The actual dimension, $\operatorname{adim}(X, t, m)$, is the dimension of the vector space of the forms in $I_{X}$ of degree $t$ vanishing at a general point $P$ with multiplicity $m$. That is,

$$
\operatorname{adim}(X, t, m)=\operatorname{dim}\left[I_{X} \cap I_{P}^{m}\right]_{t} .
$$

Next, the virtual dimension, $\operatorname{vdim}(X, t, m)$, is the dimension of the linear system of the forms of degree $t$ in $I_{X}$ minus the expected number of conditions imposed by taking $P$ with multiplicity $m$. That is,

$$
\operatorname{vdim}(X, t, m)=\operatorname{dim}\left[I_{X}\right]_{t}-\left(\begin{array}{c}
m-1+n \\
n
\end{array}\right) .
$$

Finally, the expected dimension $\operatorname{edim}(X, t, m)$ is the maximum of $\operatorname{vdim}(X, t, m)$ and 0 .

Of course, $\operatorname{adim}(X, t, m) \geq \operatorname{edim}(X, t, m) \geq \operatorname{vdim}(X, t, m)$. We say that $X$ admits an unexpected hypersurface of degree $t$ vanishing at a general point $P$ with multiplicity $m$ when $\operatorname{adim}(X, t, m)>\operatorname{edim}(X, t, m)$, i.e., when $\operatorname{adim}(X, t, m)>0$ and $\operatorname{adim}(X, t, m)>$ $\operatorname{vdim}(X, t, m)$.

The purpose of this paper is to get a better feel for when unexpected hypersurfaces are forced to occur (hence "expecting the unexpected"). We relate unexpectedness to algebraic and geometric properties (see for example Proposition 3.9 for the former and Corollary 3.5 and Theorem 6.2 for the latter) and we bring to bear methods not previously applied to unexpectedness (such as the use of generic initial ideals and partial elimination ideals) to clarify when unexpectedness can and when it cannot be expected. When it can, we also study 
to what extent it can, which we do by introducing AV sequences measuring the gap between the actual dimension and the virtual dimension (see Definition 2.8), which also allows us to frame our work in terms of persistence (i.e., how long does the gap remain positive?).

In Section 2 we define these AV sequences. Specifically, for a given subscheme $X$ of $\mathbb{P}^{n}$ and a non-negative integer $j \geq 0$, we define $A V_{X, j}: \mathbb{Z}_{>0} \rightarrow \mathbb{Z}_{\geq 0}$ where $A V_{X, j}(m):=$ $\operatorname{adim}(X, m+j, m)-\operatorname{vdim}(X, m+j, m)$. Studying the difference adim - vdim is not new (see for instance $[\mathrm{HMT}]$ ), but considering it as a sequence is novel. This sequence has interesting properties and leads to compact formulas. Sometimes we will consider the general case, but even the cases $j=0$ and $j=1$ are interesting (see Proposition 3.8 and Sections $4-6$ ).

In Section 3, for any subscheme $X$ and integer $j \geq 0$, we prove (Theorem 3.4) that the sequence $A V_{X, j}$ is actually an $O$-sequence, up to a shift. Indeed, it is the Hilbert function of the $K$-algebra $R /\left(\operatorname{gin}\left(I_{X}\right): x^{j+1}\right)$, where $\operatorname{gin}\left(I_{X}\right)$ denotes the generic initial ideal with respect to the lexicographic order. We use this fact to obtain results which ensure the nonexistence of unexpected hypersurfaces. In particular, if $X$ lies on a hyperplane or if $\operatorname{gin}\left(I_{X}\right)$ is a lex-segment ideal then $X$ does not admit any unexpected hypersurfaces of any type (Corollary 3.5 and Proposition 3.9), and hence $\operatorname{adim}(X, t, m)$ always has the expected value.

The fact that the AV sequence is actually an $O$-sequence raises many other related questions. In Section 4 we use a cohomological interpretation of the AV sequence, described in Remark 2.12, to prove that if $X$ is an irreducible, arithmetically Cohen-Macaulay (ACM) curve in $\mathbb{P}^{3}$, then $A V_{X, 1}(m)$ is unimodal and the first part is differentiable (see Theorem 4.4). Moreover, we have a great deal of experimental evidence which suggests that if $X$ is smooth then this sequence is also finite and symmetric (see Conjecture 4.1).

In Section 5 we apply a new method to study the question of unexpected hypersurfaces, namely the theory of partial elimination ideals introduced by Green [Gr]. We consider the case of a general codimension 2 complete intersection $C \subset \mathbb{P}^{n}$ and we show the non-vanishing of $\left[I_{C} \cap I_{P}^{m}\right]_{t}$ for prescribed values of $t$ and $m$ (Proposition 5.1). We apply this to the case of $n=3$ to show for $t=(a-1)(b-1)+1$ and $m=(a-1)(b-1)$, that a general complete intersection curve $C$ of type $(a, b)$ with $2<a \leq b$, admits an unexpected hypersurface of degree $t$ for multiplicity $m$ (Proposition 5.4).

In Section 6 , in the case of either a reduced equidimensional curve $C$, or the disjoint union of a finite set of points and a curve $C$, we describe how the $A V$-sequence depends only on geometric information (see Theorems 6.2 and 6.4).

In Section 7 we study how knowledge of the Hilbert function, together with certain geometric assumptions, can provide information about unexpected hypersurfaces. We restrict our attention to the case of subvarieties of $\mathbb{P}^{3}$, but we expect much more can be said in the general situation. We use results from [BGM], where maximal growth of the $h$-vector of a set of points $X$ forces the existence of a suitable curve $C$ in the base locus of some component of $I_{X}$. Then we apply some of the results of Section 6 to produce Theorem 7.8, already described above. We include several examples to show the range of things that can happen for sets with the same Hilbert function.

\section{BACKGROUND}

We now introduce the main definitions and notation we need, and begin an investigation of $A V$ sequences. We begin by recalling the notion of the $d$-Macaulay representation of a positive integer. The notion goes back to Macaulay [Mac] or earlier; see [BH] for an excellent exposition. 
Lemma 2.1 ([BH] Lemma 4.2 .6 and page 161). Let $d$ be a positive integer. Any $a \in \mathbb{Z}_{\geq 0}$ can be written uniquely in the form

$$
a=\left(\begin{array}{c}
k_{d} \\
d
\end{array}\right)+\left(\begin{array}{c}
k_{d-1} \\
d-1
\end{array}\right)+\cdots+\left(\begin{array}{c}
k_{j} \\
j
\end{array}\right)
$$

where $k_{d}>k_{d-1}>\cdots>k_{j} \geq j \geq 1$ are integers.

Given the above $d$-Macaulay representation of $a$, we define

$$
a^{\langle d\rangle}=\left(\begin{array}{c}
k_{d}+1 \\
d+1
\end{array}\right)+\left(\begin{array}{c}
k_{d-1}+1 \\
d
\end{array}\right)+\cdots+\left(\begin{array}{c}
k_{j}+1 \\
j+1
\end{array}\right)
$$

and set $0^{\langle d\rangle}=0$.

Theorem 2.2 ([BH] Theorem 4.2.10). Let $K$ be a field and let $h: \mathbb{Z}_{\geq 0} \rightarrow \mathbb{Z}_{\geq 0}$ be a numerical function. Then $h$ is the Hilbert function of some standard graded $\bar{K}$-algebra if and only if

$$
h(0)=1 \quad \text { and } \quad h(d+1) \leq h(d)^{\langle d\rangle}
$$

for all $d \geq 1$.

An infinite sequence $a_{0}, a_{1}, a_{2}, \ldots$ of non-negative integers with $a_{i}=h(i)$ for an $h$ satisfying the conditions of Theorem 2.2 is called an O-sequence. We will regard a finite sequence $a_{0}, a_{1}, a_{2}, \ldots, a_{r}$ as an infinite sequence by setting $a_{i}=0$ for $i>r$.

Hereafter, let $R=K\left[x_{0}, \ldots, x_{n}\right]$ be a polynomial ring over a field $K$. Our default assumption will be that $K$ is algebraically closed, but sometimes we will need the characteristic to be zero, and sometimes we will need only that $K$ is infinite; in these cases we will say so explicitly.

Notation 2.3. For any subvariety (or subscheme) $V \subseteq \mathbb{P}^{n}$ we write $I_{V} \subseteq R$ for the saturated ideal of $V$ and $\mathcal{I}_{V}$ for the sheaf on $\mathbb{P}^{n}$ corresponding to $I_{V}$. For a standard graded algebra $R / I$ we write $h_{R / I}(t)$ for the Hilbert function of $R / I$, i.e. $h_{R / I}(t)=\operatorname{dim}_{K}[R / I]_{t}$. When $I=I_{V}$ for some subscheme $V$, we sometimes write $h_{V}(t)$ for $h_{R / I_{V}}(t)$. We say that $V$ is arithmetically Cohen-Macaulay $(A C M)$ if $R / I_{V}$ is a Cohen-Macaulay ring.

For any integer function $h: \mathbb{Z}_{\geq 0} \rightarrow \mathbb{Z}$, the first difference $\Delta h$ is the backward difference, defined by setting $\Delta h(0)=h(0)$ and $\Delta h(t)=h(t)-h(t-1)$ for $t>0$. When $X$ is a finite set of points, it is well-known and easy to see that $h_{X}$ is strictly increasing until it becomes constant, hence there is a $j$ such that $1=h_{X}(0)<\cdots<h_{X}(j)=h_{X}(j+1)=\cdots$. We refer to $\left(\Delta h_{X}(0), \ldots, \Delta h_{X}(j)\right)$ as the $h$-vector of $X$; it is known to be a finite $O$-sequence (see, e.g., [CH, Proposition 6.3]).

Notation 2.4. Let $t \geq m$ be positive integers and let $P \in \mathbb{P}^{n}$ be a general point. Given $X \subset \mathbb{P}^{n}$ a subscheme, we set

$$
\begin{array}{ll}
\operatorname{adim}(X, t, m)=\operatorname{adim}\left(I_{X}, t, m\right)=\operatorname{dim}\left[I_{X} \cap I_{P}^{m}\right]_{t} & \text { (the actual dimension), } \\
\operatorname{vdim}(X, t, m)=\operatorname{vdim}\left(I_{X}, t, m\right)=\operatorname{dim}\left[I_{X}\right]_{t}-\left(\begin{array}{c}
m+n-1 \\
n
\end{array}\right) & \text { (the virtual dimension), } \\
\operatorname{edim}(X, t, m)=\operatorname{edim}\left(I_{X}, t, m\right)=\max \{0, \operatorname{vdim}(X, t, m)\} & \text { (the expected dimension). }
\end{array}
$$

Note that we always have

$$
\operatorname{adim}(X, t, m) \geq \operatorname{edim}(X, t, m) \geq \operatorname{vdim}(X, t, m) .
$$


Definition 2.5. If $\operatorname{adim}(X, t, m)>\operatorname{edim}(X, t, m)$, we say that $X$ admits an unexpected hypersurface of degree $t$ for multiplicity $m$. (In this case, note that $\operatorname{adim}(X, t, m)>0$, hence $t \geq m$.) If $X \subset \mathbb{P}^{2}$ is a finite set of points which admits an unexpected hypersurface of degree $t$ for multiplicity $m=t-1$, then following [CHMN] we say simply that $X$ admits an unexpected curve of degree $t$.

Remark 2.6. An equivalent condition for $X$ to admit an unexpected hypersurface of degree $t$ for multiplicity $m$ is $\operatorname{adim}(X, t, m)>0$ and $\operatorname{adim}(X, t, m)>\operatorname{vdim}(X, t, m)$.

Remark 2.7. Any hypersurface of degree $t$ with an isolated singularity of multiplicity $t$ must be a cone (by Bezout's theorem). Thus $\operatorname{adim}(X, t, t)$ is the dimension of the vector space of cones over $X$ of degree $t$ with vertex at $P$. If $\operatorname{adim}(X, t, t)>\operatorname{edim}(X, t, t)$, we say that $X$ admits an unexpected cone of degree $t$. See [HMNT, CM, HMT] for more on unexpected cones. In particular, if $X$ has codimension two and is reduced, equidimensional and non-degenerate then the cone $S_{P}$ over $X$ with vertex $P$ is an unexpected cone of degree $t=\operatorname{deg} X$ ([HMNT] Proposition 2.4).

Definition 2.8. Let $X \subset \mathbb{P}^{n}$ be a closed subscheme. Fixing a non-negative integer $j$, we define the sequence $A V_{X, j}$ as follows:

$$
A V_{X, j}(m)=\operatorname{adim}(X, m+j, m)-\operatorname{vdim}(X, m+j, m), \quad m \geq 1 .
$$

Remark 2.9. Rephrasing Remark 2.6, if $\operatorname{adim}(X, t, m)>0$ then $X$ admits an unexpected hypersurface of degree $t$ for multiplicity $m$ if and only if $A V_{X, j}(m)>0$ for $j=t-m$.

Notation 2.10. Let $P \in \mathbb{P}^{n}$ be a general point, with defining ideal $I_{P}$. We will denote the scheme defined by $I_{P}^{m}$ in $\mathbb{P}^{n}$ by $P^{m}$. We will sometimes consider the hyperplane section of $P^{m}$ by a hyperplane $H$ containing $P$, and we will denote the corresponding subscheme of $H$ by $m P$, thus $m P=P^{m} \cap H$.

We now give an interpretation of the sequence $A V_{X, j}$. Notice that, in the following lemma, the ideal in the dimension of the quotient on the right changes with $m$.

Lemma 2.11. Let $X \subset \mathbb{P}^{n}$ be a subscheme. Then

$$
A V_{X, j}(m)=\operatorname{dim}\left[R /\left(I_{X}+I_{P}^{m}\right)\right]_{m+j} .
$$

Proof. Set $t:=m+j$. From the short exact sequence

$$
0 \rightarrow R /\left(I_{X} \cap I_{P}^{m}\right) \rightarrow R / I_{X} \oplus R / I_{P}^{m} \rightarrow R /\left(I_{X}+I_{P}^{m}\right) \rightarrow 0
$$

we get the relation between the dimension of the modules in degree $t$ $\operatorname{dim}[R]_{t}-\operatorname{adim}(X, t, m)-\operatorname{dim}[R]_{t}+\operatorname{dim}\left[I_{X}\right]_{t}-\operatorname{dim}[R]_{t}+\operatorname{dim}\left[I_{P}^{m}\right]_{t}+\operatorname{dim}\left[R /\left(I_{X}+I_{P}^{m}\right)\right]_{t}=0$.

Therefore

$$
\begin{aligned}
\operatorname{dim}\left[R /\left(I_{X}+I_{P}^{m}\right)\right]_{t} & =\operatorname{adim}(X, t, m)-\operatorname{dim}\left[I_{X}\right]_{t}+\operatorname{dim}[R]_{t}-\operatorname{dim}\left[I_{P}^{m}\right]_{t}= \\
& =\operatorname{adim}(X, t, m)-\operatorname{dim}\left[I_{X}\right]_{t}+h_{P^{m}}(t) .
\end{aligned}
$$

Since $t \geq m$ the Hilbert function of the fat point $P^{m}$ in degree $t$ is $h_{P^{m}}(t)=\left(\begin{array}{c}m+n-1 \\ n\end{array}\right)$. reaches the degree $\operatorname{deg}\left(P^{m}\right)$ in degree $m-1$.) So we get

$$
\operatorname{dim}\left[R /\left(I_{X}+I_{P}^{m}\right)\right]_{t}=\operatorname{adim}(X, t, m)-\operatorname{vdim}(X, t, m)
$$

as desired. 
Remark 2.12. We can also give a cohomological interpretation for the sequence $A V_{X, j}(m)$. Let $X$ be an ACM subscheme in $\mathbb{P}^{n}$ of dimension $\geq 1$. Assume $m \geq 0$ and $j \geq 0$. Consider the exact sequence of sheaves

$$
0 \rightarrow \mathcal{I}_{X \cup P^{m}} \rightarrow \mathcal{I}_{X} \rightarrow \mathcal{O}_{P^{m}} \rightarrow 0
$$

Twisting by $m+j$ and taking cohomology gives the exact sequence

$$
0 \rightarrow\left[I_{X \cup P^{m}}\right]_{m+j} \rightarrow\left[I_{X}\right]_{m+j} \rightarrow H^{0}\left(\mathcal{O}_{P^{m}}(m+j)\right) \rightarrow H^{1}\left(\mathcal{I}_{X \cup P^{m}}(m+j)\right) \rightarrow 0 .
$$

(Exactness on the right is because $X$ is ACM of dimension $\geq 1$; see [Mi, pp. 9-11].) This gives

$$
h^{1}\left(\mathcal{I}_{X \cup P^{m}}(m+j)\right)=\left(\begin{array}{c}
(m-1)+n \\
n
\end{array}\right)-\operatorname{dim}\left[I_{X}\right]_{m+j}+\operatorname{dim}\left[I_{X \cup P^{m}}\right]_{m+j}=A V_{X, j}(m) .
$$

Remark 2.13. Given a subscheme $X \subset \mathbb{P}^{n}$, it is natural to ask about the persistence of the unexpectedness imposed by $X$. For example, in [HMNT, Corollary 2.12], it is shown that a nondegenerate curve $C \subset \mathbb{P}^{3}$ of degree $d=\operatorname{deg} C$ admits an unexpected hypersurface of degree $t$ for multiplicity $t$ at a general point for all $t \geq d$. Thus fixing $0=j=t-m$, and fixing $C$, we have the persistence of unexpectedness as long as $t \geq d$.

Many of the results in this paper give formulas for the sequences $A V_{X, j}(m)$. Leaving aside the issue of whether $\operatorname{adim}(X, t, m)>0$, this sequence can be interpreted both as a measure of unexpectedness (how much bigger is the actual dimension than what one would expect?) and as a measure of persistence (how long is $A V_{X, j}(m)$ positive?). The fact that these sequences are represented by simple formulas, as we will see in the coming sections, is a pleasant bonus.

\section{Generic initial ideals and unexpeCtedness}

In this section we relate the study of unexpected hypersurfaces of a subscheme $X \subseteq \mathbb{P}^{n}$ to the generic initial ideal of $I_{X}$ with respect to the lexicographic order. Then, we prove that the $A V_{X, j}$ sequence, up to a shift, is an $O$-sequence. As a consequence of this result we are able to ensure the non-existence of unexpected hypersurfaces in several cases.

Let $R=K\left[x_{0}, x_{1}, \ldots, x_{n}\right]$ be a standard graded polynomial ring. In this section we only require $K$ to be infinite. We assume the monomials of $R$ are ordered by $>_{\text {lex }}$, the lexicographic monomial order which satisfies $x_{0}>x_{1}>\cdots>x_{n}$. We recall that a set $M \subseteq R$ of monomials is a lex-segment if the monomials have the same degree and they satisfy the condition that whenever $u, v$ are monomials with $u \geq v$ and $v \in M$, then $u \in M[\mathrm{~V}]$. It is convenient to also refer to a vector subspace $W \subseteq R$ as a lex-segment if $W$ is spanned by a lex-segment in the previous sense. We also recall that a homogeneous ideal $I \subseteq R$ is a lex-segment ideal if for each degree $d$ the the homogeneous component $I_{d}$ of $I$ of degree $d$ is a lex segment $[\mathrm{V}]$; see also $[\mathrm{Hu}]$.

For a graded ideal $I \subseteq R$, we will denote by gin $(I)$ the generic initial ideal of $I$ with respect to the monomial order $>_{\text {lex }}$. For an introduction to generic initial ideals, see for instance [Gr] and Section 15.9 in [E]. The next lemma relates the actual and virtual dimensions of a scheme in terms of the generic initial ideal of its ideal.

Lemma 3.1. Let $X \subseteq \mathbb{P}^{n}$ be a subscheme. For any non-negative integers $t$ and $m$, we have

(i) $\operatorname{adim}(X, t, m)=\operatorname{dim}\left[\operatorname{gin}\left(I_{X}\right) \cap I_{Q}^{m}\right]_{t}$, where $Q=(1,0, \ldots, 0)$.

(ii) $\operatorname{vdim}(X, t, m)=\operatorname{vdim}\left(\operatorname{gin}\left(I_{X}\right), t, m\right)$. 
Proof. (i) Let $X^{\prime}$ be the image of $X$ under a general linear change of variables, so gin $\left(I_{X}\right)=$ $\operatorname{in}\left(I_{X^{\prime}}\right)$ and so the point $Q$ is general for $X^{\prime}$. Moreover, if $\mu_{1}, \mu_{2}$ are monomials of the same degree with $\mu_{1} \in\left[I_{Q}^{m}\right]_{t}$ and $\mu_{1}>_{\text {lex }} \mu_{2}$, then $\mu_{2} \in I_{Q}^{m}$, hence $\operatorname{in}\left(I_{X^{\prime}} \cap I_{Q}^{m}\right)=\operatorname{in}\left(I_{X^{\prime}}\right) \cap I_{Q}^{m}$. (To see this note that both sides of the equality are monomial ideals, and that $\operatorname{in}\left(I_{X^{\prime}} \cap I_{Q}^{m}\right) \subseteq$ $\operatorname{in}\left(I_{X^{\prime}}\right) \cap I_{Q}^{m}$ is clear. So suppose that $w \in \operatorname{in}\left(I_{X^{\prime}}\right) \cap I_{Q}^{m}$ is a monomial. Then there is a form $W \in I_{X^{\prime}}$ with $w=\operatorname{in}(W)$. It follows that the other terms of $W$ have lex order less than $w$, so each is in $I_{Q}^{m}$, hence $W \in I_{Q}^{m}$, and we have $W \in I_{X^{\prime}} \cap I_{Q}^{m}$ so $w \in \operatorname{in}\left(I_{X^{\prime}} \cap I_{Q}^{m}\right)$ giving $\operatorname{in}\left(I_{X^{\prime}} \cap I_{Q}^{m}\right) \supseteq \operatorname{in}\left(I_{X^{\prime}}\right) \cap I_{Q}^{m}$. $)$ Thus we have $\operatorname{adim}(X, t, m)=\operatorname{dim}\left[I_{X} \cap I_{P}^{m}\right]_{t}=\operatorname{dim}\left[I_{X^{\prime}} \cap I_{Q}^{m}\right]_{t}=$ $\operatorname{dim}\left[\operatorname{in}\left(I_{X^{\prime}} \cap I_{Q}^{m}\right)\right]_{t}=\operatorname{dim}\left[\operatorname{in}\left(I_{X^{\prime}}\right) \cap I_{Q}^{m}\right]_{t}=\operatorname{dim}\left[\operatorname{gin}\left(I_{X}\right) \cap I_{Q}^{m}\right]_{t}$.

(ii) This is a consequence of $h_{X}=h_{R /\left(\operatorname{gin}\left(I_{X}\right)\right)}$.

Remark 3.2. Let $I=\oplus_{k} I_{k}$ be a homogeneous ideal in $R$. Then we make the following definition (see [Gr, Definition 6.1]):

$$
\widetilde{K}_{d}(I)=\bigoplus_{k}\left[I \cap I_{Q}^{k-d}\right]_{k}
$$

where $Q=(1,0, \ldots, 0)$, so $\widetilde{K}_{d}(I)$ is a graded module over $K\left[x_{1}, \ldots, x_{n}\right]$. In particular, $\widetilde{K}_{0}(I)$ is an ideal: it is obtained from $I$ by eliminating the variable $x_{0}$. Geometrically, it corresponds to the linear space of the cones having vertex at $Q$. Then Lemma 3.1 (i) can be rephrased as follows:

$$
\operatorname{dim}\left(I_{X} \cap I_{P}^{m}\right)_{t}=\operatorname{dim}\left[\widetilde{K}_{t-m}\left(\operatorname{gin}\left(I_{X}\right)\right)\right]_{t} .
$$

Example 3.3. Assume $\operatorname{char}(K)=0$. Here, given only the Hilbert function $h_{X}$ of a set of points $X \subset \mathbb{P}^{2}$, we show how information about $\operatorname{gin}\left(I_{X}\right)$ relates to whether or not $X$ has an unexpected curve. So let $X \subseteq \mathbb{P}^{2}$ be a set of 13 points with $h_{X}=(1,3,6,10,12,13,13, \ldots)$, so $X$ lies on three independent quartics but no cubics (such examples exist, as we see later in this example). Then $\operatorname{dim}\left[I_{X}\right]_{6}=15$, so vdim $(X, 6,5)=0$, hence $X$ admits an unexpected curve of degree 6 if and only if $\operatorname{adim}(X, 6,5)>0$. From Lemma $3.1(\mathrm{i}), \operatorname{adim}(X, 6,5)>0$ if and only if $\operatorname{dim}\left[\operatorname{gin}\left(I_{X}\right) \cap I_{Q}^{5}\right]_{6}>0$. In this case, the ideal defining the point $Q$ is $I_{Q}=(y, z) \subseteq K[x, y, z]$, so the monomials of degree 6 contained in $\left[I_{Q}^{5}\right]_{6}$ are exactly those lexicographically less than or equal to $x y^{5}$. But (in characteristic 0) generic initial ideals are (by [CaS]) strongly stable (see [AL] for the definition and properties of strong stability), hence if any monomial of degree 6 less than or equal to $x y^{5}$ is in $\operatorname{gin}\left(I_{X}\right)$, then $x y^{5}$ is in $\operatorname{gin}\left(I_{X}\right)$ too. Thus $X$ admits an unexpected curve of degree 6 if and only if $x y^{5} \in \operatorname{gin}\left(I_{X}\right)$.

Now we determine all the monomials in $\left[\operatorname{gin}\left(I_{X}\right)\right]_{\leq 6}$, assuming that $X$ admits no unexpected curves in degrees strictly lower than 6 . With this assumption and using the fact that unexpected curves have degree at least 4 [A, FGST], from Lemma 3.1(i) we have

- $\left[\operatorname{gin}\left(I_{X}\right) \cap I_{Q}^{3}\right]_{4}=\left[\operatorname{gin}\left(I_{X}\right) \cap I_{Q}^{4}\right]_{5}=(0)$, so if $x^{a} y^{b} z^{c} \in\left[\operatorname{gin}\left(I_{X}\right)\right]_{\leq 6}$ then $a \geq 2$, and

- $\operatorname{edim}(X, 4,2)=\operatorname{adim}(X, 4,2)=0$ (by Bertini's Theorem), hence $\left[\operatorname{gin}\left(I_{X}\right) \cap I_{Q}^{2}\right]_{4}=(0)$, so if $x^{a} y^{b} z^{c} \in\left[\operatorname{gin}\left(I_{X}\right)\right]_{4}$ then $a \geq 3$.

Collecting this information, we get only one strongly stable ideal through degree 5 (with the given Hilbert function), that is $\left(x^{4}, x^{3} y, x^{3} z, x^{2} y^{3}, x^{2} y^{2} z\right)$. So, $\left[\operatorname{gin}\left(I_{X}\right)\right]_{\leq 5}$ is a lex segment.

Taking generators up to degree 6 of the lex-segment ideal with Hilbert function $h_{X}$ we get

$$
L=\left(x^{4}, x^{3} y, x^{3} z, x^{2} y^{3}, x^{2} y^{2} z, x^{2} y z^{3}, x^{2} z^{4}\right),
$$


thus $X$ has an unexpected curve of degree 6 with a general multiple point of multiplicity 5 if and only if the component $\left[\operatorname{gin}\left(I_{X}\right)\right]_{6}$ fails to be equal to the degree 6 component of the lex-segment ideal with the same Hilbert function.

As promised, we now show that sets $X$ do arise. We first give an example of a set $X$ of 13 points with no unexpected curves of degree 6 or less. We get 12 points of the points of $X$ as the complete intersection of a general cubic and quartic; add to this a general point $Q$ to obtain $X$. It is easy to see that $X$ has the Hilbert function as specified above, and by direct computation with Macaulay2 [GS], we find that $X$ has no unexpected curves of degree 6 or less. In this case, we find from Macaulay2 that $\operatorname{gin}\left(I_{X}\right)=$ $\left(x^{4}, x^{3} y, x^{3} z, x^{2} y^{3}, x^{2} y^{2} z, x^{2} y z^{3}, x^{2} z^{4}, x y^{6}, x y^{5} z, x y^{4} z^{3}, x y^{3} z^{5}, x y^{2} z^{7}, x y z^{9}, x z^{11}, y^{13}\right)$, hence $x y^{5}$, as claimed, does not occur.

We now give two examples of an $X$ with the specified Hilbert function which do have unexpected sextics. Returning to the specfied Hilbert function, we see that two monomials of degree 6 are needed in the minimal set of generators of gin $\left(I_{X}\right)$. If $X$ admits an unexpected curve of degree 6 with a general multiple point of multiplicity 5 , we have already noticed that $x y^{5} \in \operatorname{gin} I_{X}$. Thus, the strongly stable property forces $\left[\operatorname{gin}\left(I_{X}\right)\right]_{\leq 6}$ to be either

$$
\mathcal{G}_{1}=\left(x^{4}, x^{3} y, x^{3} z, x^{2} y^{3}, x^{2} y^{2} z, x^{2} y z^{3}, x y^{5}\right)
$$

or

$$
\mathcal{G}_{2}=\left(x^{4}, x^{3} y, x^{3} z, x^{2} y^{3}, x^{2} y^{2} z, x y^{5}, x y^{4} z\right) .
$$

By direct computation we see that a set of points $X$ with $\left[\operatorname{gin}\left(I_{X}\right)\right]_{\leq 6}=\mathcal{G}_{2}$ would have $\operatorname{adim}(X, 6,5)=2$, but by [CHMN, Corollary 5.5] this would mean $X$ has an unexpected quintic, contrary to our assumption that $X$ admits no unexpected curves in degrees strictly lower than 6 . Thus $\left[\operatorname{gin}\left(I_{X}\right)\right]_{\leq 6}=\mathcal{G}_{2}$ cannot occur if $X$ is a reduced set of 13 points.

However, $\left[\operatorname{gin}\left(I_{X}\right)\right]_{\leq 6}=\mathcal{G}_{1}$ can occur; we give two examples. Specifically, the following two sets of points $X_{1}$ and $X_{2}$ have $h_{X_{1}}=h_{X_{2}}=h_{X}$ and $\left[\operatorname{gin}\left(I_{X_{i}}\right)\right]_{\leq 6}=\mathcal{G}_{1}$. The lines (see Figure $1)$ dual to the points $X_{1}$ give what [DMO] refers to as a $(1,1)$ tic-tac-toe arrangement:

$$
\begin{gathered}
X_{1}:=\{(1,0,0),(0,1,0),(0,0,1),(1,1,0),(0,1,1),(1,0,1),(-1,1,0),(0,-1,1),(-1,0,1), \\
(1,1,1),(-1,1,1),(-1,1,-1),(1,1,-1)\} .
\end{gathered}
$$

The ideal defining $X_{1}$ is

$$
I_{X_{1}}=\left(y^{3} z-y z^{3}, x^{3} z-x z^{3}, x^{3} y-x y^{3}\right) .
$$

The second set of points is

$$
\begin{gathered}
X_{2}:=\{(1,0,0),(0,1,0),(0,0,1),(1,1,0),(0,1,1),(1,0,1),(-1,1,0),(0,-1,1),(-1,0,1), \\
(2,1,1),(-2,1,1),(-2,1,-1),(2,1,-1)\} ;
\end{gathered}
$$

it is defined by the ideal

$$
I_{X_{2}}=\left(y^{3} z-y z^{3}, x^{3} z-x z^{3}-3 x y^{2} z, x^{3} y-x y^{3}-3 x y z^{2}\right) .
$$

(The lines dual to $X_{2}$ are shown in Figure 2.)

We now show that the sequence $A V_{X, j}$ is the Hilbert function of a fixed standard graded algebra; since $A V_{X, j}(m)$ is defined for $m \geq 1$ we need to shift it by 1 . 


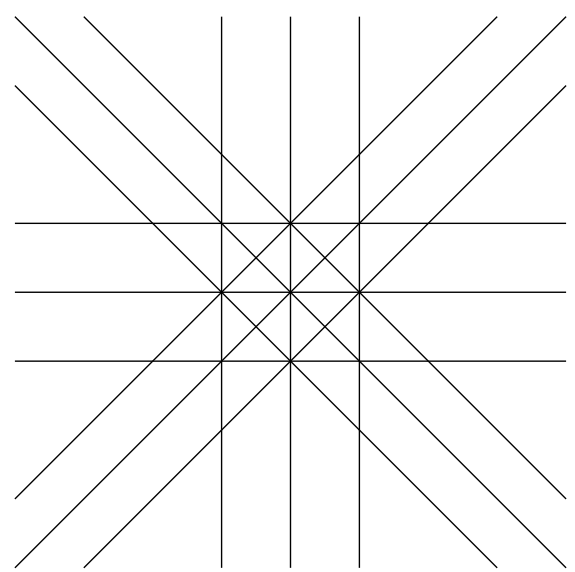

FiguRE 1. A sketch of the line configuration dual to the points of $X_{1}$ from Example 3.3. (The line at infinity, corresponding to the point $(0,0,1)$, is not shown).

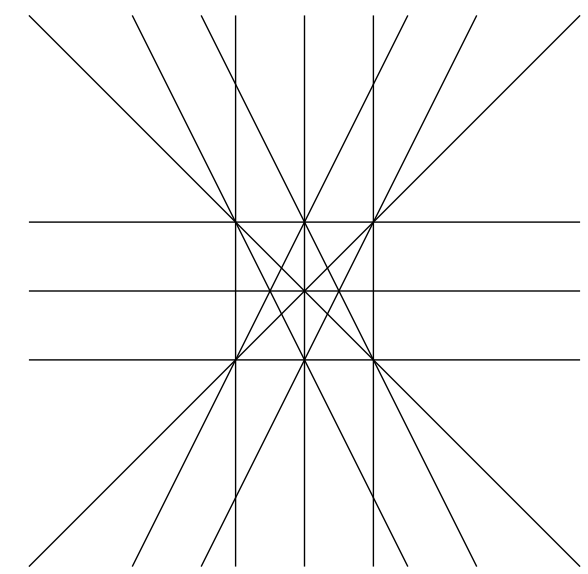

Figure 2. A sketch of the line configuration dual to the points of $X_{2}$ from Example 3.3. (The line at infinity, corresponding to the point $(0,0,1)$, is not shown).

Theorem 3.4. For any non-negative integer $j$, the sequence $A V_{X, j}$ shifted to the left by 1 is an $O$-sequence. In particular, setting $J:=\operatorname{gin}\left(I_{X}\right): x_{0}^{j+1}$, the sequence $A V_{X, j}$ shifted to the left by 1 coincides with the Hilbert function of $R / J$, i.e.,

$$
A V_{X, j}(d+1)=h_{R / J}(d), d \geq 0 .
$$

Proof. Set $\mathfrak{q}=\left(x_{1}, \cdots, x_{n}\right)$ the ideal defining the point $Q=(1,0, \ldots, 0)$. For any $m \geq 1$ and for any non-negative integer $j$, from Lemma 2.11, reasoning as in the proof of Lemma 3.1, we have

$$
A V_{X, j}(m)=\operatorname{dim}\left[R /\left(I_{X}+I_{P}^{m}\right)\right]_{m+j}=\operatorname{dim}\left[R /\left(\operatorname{gin}\left(I_{X}\right)+\mathfrak{q}^{m}\right)\right]_{m+j} .
$$

For a monomial ideal $T$, it is easy to show that we can write $[T]_{m+j}$ as the following direct sum (and so the summands have only 0 in common):

$$
[T]_{m+j}=\left[x_{0}^{j+1} \cdot\left(T: x_{0}^{j+1}\right)\right]_{m+j} \bigoplus\left[T \cap \mathfrak{q}^{m}\right]_{m+j} .
$$


In particular,

$$
[R]_{m+j}=\left[x_{0}^{j+1} \cdot\left(R: x_{0}^{j+1}\right)\right]_{m+j} \bigoplus\left[R \cap \mathfrak{q}^{m}\right]_{m+j}=\left[x_{0}^{j+1} R\right]_{m+j} \bigoplus\left[\mathfrak{q}^{m}\right]_{m+j},
$$

so $\operatorname{dim}\left[R / \mathfrak{q}^{m}\right]_{m+j}=\operatorname{dim}\left[x_{0}^{j+1} R\right]_{m+j}$. Similarly,

$$
\begin{aligned}
{\left[\operatorname{gin}\left(I_{X}\right)+\mathfrak{q}^{m}\right]_{m+j} } & =\left[x_{0}^{j+1} \cdot\left(\left(\operatorname{gin}\left(I_{X}\right)+\mathfrak{q}^{m}\right): x_{0}^{j+1}\right)\right]_{m+j} \bigoplus\left[\left(\operatorname{gin}\left(I_{X}\right)+\mathfrak{q}^{m}\right) \cap \mathfrak{q}^{m}\right]_{m+j} \\
& =\left[x_{0}^{j+1} \cdot\left(\operatorname{gin}\left(I_{X}\right): x_{0}^{j+1}\right)\right]_{m+j} \bigoplus\left[\mathfrak{q}^{m}\right]_{m+j},
\end{aligned}
$$

so $\operatorname{dim}\left[\left(\operatorname{gin}\left(I_{X}\right)+\mathfrak{q}^{m}\right) / \mathfrak{q}^{m}\right]_{m+j}=\operatorname{dim}\left[x_{0}^{j+1} \cdot\left(\operatorname{gin}\left(I_{X}\right): x_{0}^{j+1}\right)\right]_{m+j}$. Thus

$$
\begin{aligned}
\operatorname{dim}\left[\frac{R}{\operatorname{gin}\left(I_{X}\right)+\mathfrak{q}^{m}}\right]_{m+j} & =\operatorname{dim}\left[\frac{R / \mathfrak{q}^{m}}{\left(\operatorname{gin}\left(I_{X}\right)+\mathfrak{q}^{m}\right) / \mathfrak{q}^{m}}\right]_{m+j} \\
& =\operatorname{dim}\left[R / \mathfrak{q}^{m}\right]_{m+j}-\operatorname{dim}\left[\left(\operatorname{gin}\left(I_{X}\right)+\mathfrak{q}^{m}\right) / \mathfrak{q}^{m}\right]_{m+j} \\
& =\operatorname{dim}\left[x_{0}^{j+1} R\right]_{m+j}-\operatorname{dim}\left[x_{0}^{j+1} \cdot\left(\operatorname{gin}\left(I_{X}\right): x_{0}^{j+1}\right)\right]_{m+j} \\
& =\operatorname{dim}[R]_{m-1}-\operatorname{dim}\left[\operatorname{gin}\left(I_{X}\right): x_{0}^{j+1}\right]_{m-1} \\
& =\operatorname{dim}\left[R /\left(\operatorname{gin}\left(I_{X}\right): x_{0}^{j+1}\right)\right]_{m-1} .
\end{aligned}
$$

As a consequence of Theorem 3.4 we get a new criterion (in this case, geometric) for the non-existence of unexpected hypersurfaces.

Corollary 3.5. If $X$ is a reduced subscheme of $\mathbb{P}^{n}$ contained in a hypersurface of degree $d+1 \geq 1$, then for any $t \geq d+m$ and $m \geq 1$ we have

$$
\operatorname{adim}(X, t, m)=\operatorname{vdim}(X, t, m) .
$$

In particular, if $X$ is degenerate (meaning, $X$ is contained in a hyperplane, or, equivalently, $d=0$ ), then $X$ admits no unexpected hypersurfaces for multiplicity $m$ in degrees $t \geq m$, and hence no unexpected hypersurfaces of any kind.

Proof. Since $I_{X}$ has an element of degree $d+1, x_{0}^{d+1} \in \operatorname{gin}\left(I_{X}\right)$. This implies $1 \in\left(\operatorname{gin}\left(I_{X}\right)\right.$ : $x_{0}^{t-m+1}$ ) when $t \geq d+m, m \geq 0$. From Theorem 3.4 and Definition 2.8, for $t=m+j, j \geq$ $d, m \geq 1$, we have $0=A V_{X, j}(m)=\operatorname{adim}(X, t, m)-\operatorname{vdim}(X, t, m)$. The last part, about unexpected hypersurfaces, follows from Definition 2.5.

The next corollary compares the sequences $A V_{X, j}(m)$ and $A V_{X, j+1}(m)$ for a subscheme $X$.

Corollary 3.6. Let $X$ be a subscheme of $\mathbb{P}^{n}$. Then

$$
A V_{X, j}(m) \geq A V_{X, j+1}(m) .
$$

Proof. From Theorem 3.4 we have, for any $i$,

$$
A V_{X, i}(m)=\operatorname{dim}\left[R /\left(\operatorname{gin}\left(I_{X}\right): x_{0}^{i+1}\right)\right]_{m-1} .
$$

So the statement is equivalent to proving that

$$
\operatorname{dim}\left[\operatorname{gin}\left(I_{X}\right): x_{0}^{j+1}\right]_{m-1} \leq \operatorname{dim}\left[\operatorname{gin}\left(I_{X}\right): x_{0}^{j+2}\right]_{m-1},
$$


and this is trivial since we always have $\operatorname{gin}\left(I_{X}\right): x_{0}^{j+1} \subseteq \operatorname{gin}\left(I_{X}\right): x_{0}^{j+2}$.

Lemma 3.7. Let $P$ be a general point in $\mathbb{P}^{n}$. Let $Y_{1}$ be the zero-dimensional subscheme of $\mathbb{P}^{n}$ defined by $I_{P}^{m}$ and let $Y_{2}$ be the subscheme of $Y_{1}$ defined by $I_{P}^{m-1}$. Fix a positive integer $t \geq m-1$ and consider the component $\left[I_{X}\right]_{t}$, where $X$ is some subvariety of $\mathbb{P}^{n}$. If $Y_{1}$ imposes $\left(\begin{array}{c}m-1+n \\ n\end{array}\right)$ independent conditions on $\left[I_{X}\right]_{t}$ then $Y_{2}$ imposes $\left(\begin{array}{c}m-2+n \\ n\end{array}\right)$ independent conditions on $\left[I_{X}\right]_{t}$.

Proof. We know that $\operatorname{deg}\left(Y_{1}\right)=\left(\begin{array}{c}m-1+n \\ n\end{array}\right)$ and $\operatorname{deg}\left(Y_{2}\right)=\left(\begin{array}{c}m-2+n \\ n\end{array}\right)$, and that $Y_{1}$ and $Y_{2}$ impose independent conditions on $[R]_{t}$ since $t \geq m-1$ (by a regularity argument). In particular we know that

$$
\operatorname{dim}\left[I_{Y_{1}}\right]_{t}=\operatorname{dim}[R]_{t}-\left(\begin{array}{c}
m-1+n \\
n
\end{array}\right) \quad \text { and } \quad \operatorname{dim}\left[I_{Y_{2}}\right]_{t}=\operatorname{dim}[R]_{t}-\left(\begin{array}{c}
m-2+n \\
n
\end{array}\right) .
$$

Consider first the exact sequence

$$
0 \rightarrow I_{Y_{1}} \rightarrow I_{Y_{2}} \rightarrow A \rightarrow 0
$$

where $A$ is the quotient, and is supported on $P$. We have

$$
\operatorname{dim}[A]_{t}=\left(\begin{array}{c}
m-1+n \\
n
\end{array}\right)-\left(\begin{array}{c}
m-2+n \\
n
\end{array}\right)=\left(\begin{array}{c}
m-2+n \\
n-1
\end{array}\right) .
$$

Let $\mathcal{A}$ be the sheafification of $A$. From the above exact sequence and the fact that $t \geq$ $m-1\left(\right.$ so $\left.h^{1}\left(\mathcal{I}_{P}^{m}(t)\right)=h^{1}\left(\mathcal{I}_{Y_{1}}(t)\right)=0\right)$ and the fact that $I_{Y_{1}}$ and $I_{Y_{2}}$ are saturated, we get $h^{0}(\mathcal{A}(t))=\operatorname{dim}[A]_{t}$.

Remembering that $X$ and $P$ are disjoint, consider the exact sequence of sheaves

$$
0 \rightarrow \mathcal{I}_{X \cup Y_{1}}(t) \rightarrow \mathcal{I}_{X \cup Y_{2}}(t) \rightarrow \mathcal{A}(t) \rightarrow 0 .
$$

Taking cohomology we obtain

$$
0 \rightarrow\left[I_{X} \cap I_{P}^{m}\right]_{t} \rightarrow\left[I_{X} \cap I_{P}^{m-1}\right]_{t} \rightarrow[A]_{t} \rightarrow \cdots
$$

Hence

$$
\begin{aligned}
\operatorname{dim}\left[I_{X} \cap I_{Y_{2}}\right]_{t} & =\operatorname{dim}\left[I_{X} \cap I_{P}^{m-1}\right]_{t} \\
& \leq \operatorname{dim}\left[I_{X} \cap I_{P}^{m}\right]_{t}+\operatorname{dim}[A]_{t} \\
& =\operatorname{dim}\left[I_{X}\right]_{t}-\left(\begin{array}{c}
m-1+n \\
n
\end{array}\right)+\left(\begin{array}{c}
m-2+n \\
n-1
\end{array}\right) \\
& =\operatorname{dim}\left[I_{X}\right]_{t}-\left(\begin{array}{c}
m-2+n \\
n
\end{array}\right) .
\end{aligned}
$$

But $Y_{2}$ cannot impose more conditions in degree $t$ than its degree, so we must have equality.

Like Corollary 3.5, the next result gives a criterion (again basically geometric), based on one single piece of information, for a subvariety $X \subset \mathbb{P}^{n}$ to admit no unexpected hypersurfaces of any degree or multiplicity at a general point.

Proposition 3.8. Let $X \subset \mathbb{P}^{n}$ be a subscheme. Let $\alpha:=\alpha\left(I_{X}\right)$ be the least degree $t$ such that $\left[I_{X}\right]_{t} \neq 0$. If $A V_{X, 0}(\alpha)=0$, then $X$ does not admit unexpected hypersurfaces, for any degree and multiplicity at a general point. 
Proof. By Remark 2.6, $X$ fails to admit an unexpected hypersurface of degree $t$ for multiplicity $m$ if and only if $T_{t, m}=\min \left(\operatorname{adim}(X, t, m), A V_{X, t-m}(m)\right)=0$. Consider the table $T$ of values of $T_{t, m}$ for $t, m \geq 1$. It suffices to show we always have $T_{t, m}=0$. We will divide the table into three regions as follows:

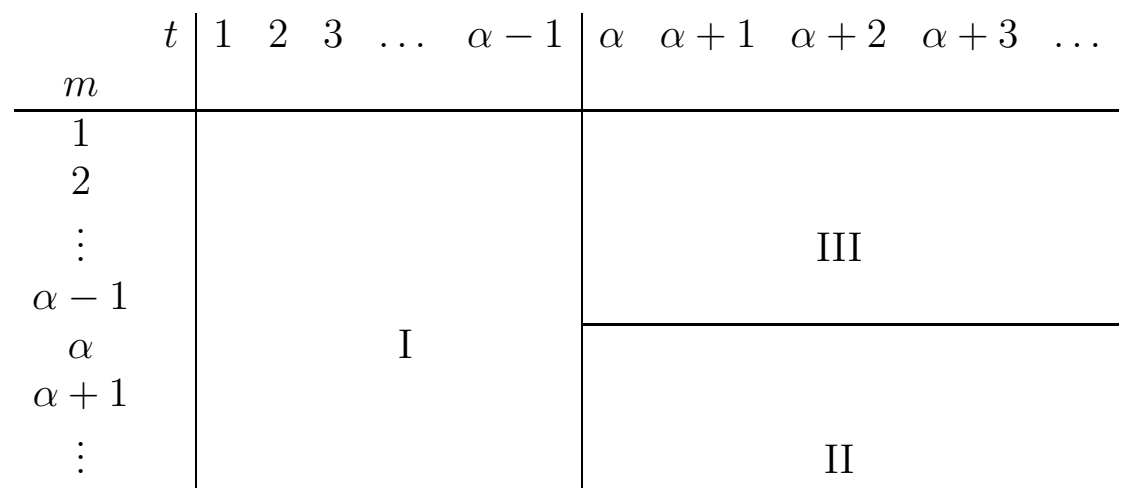

As always, $P$ denotes a general point.

For $t<\alpha$ it is clear that $T_{t, m}=\operatorname{adim}(X, t, m)=0$. This takes care of region $\mathrm{I}$.

We now show that all entries in region II are 0 . The condition $A V_{X, 0}(\alpha)=0$ gives us that $T_{\alpha, \alpha}=0$; this the top left point of region II. It also gives us that $Y_{1}$, defined by $I_{P}^{\alpha}$, imposes $\left(\begin{array}{c}\alpha-1+n \\ n\end{array}\right)$ independent conditions on $\left[I_{X}\right]_{\alpha}$. But then by Corollary 3.6 we have $T_{j+\alpha, \alpha}=A V_{X, j}(\alpha)=0$ for all $j \geq 0$ (this gives the top row of region II).

In the portion below the main diagonal of region II we have $m>t$, so $T_{t, m}=\operatorname{adim}(X, t, m)$ is 0 , since there there can be no hypersurfaces with degree $t$ and multiplicity $m$ (unexpected or not). For the portion on and above the main diagonal of region II, we apply Theorem 3.4, which says that $A V_{X, j}(m)$ is an $O$-sequence. Recall that the top row of region II gives us $A V_{X, j}(\alpha)=0$ for $j \geq 0$. Then it follows for $i>0$ that $T_{\alpha+i+j, \alpha+i}=A V_{X, j}(\alpha+i)=0$ as well, since a Hilbert function that attains a value 0 cannot subsequently become non-zero. Thus starting from each entry on the top row of region II (all of which are 0), the entries descending diagonally and to the right are all 0 . Thus all entries in region II above the main diagonal are also 0.

We now show all entries in region III are 0. Because $A V_{X, j}(\alpha)=0$, the scheme $Y_{1}$ defined by the (saturated) ideal $I_{P}^{\alpha}$ imposes independent conditions on $\left[I_{X}\right]_{t}$ for all $t \geq \alpha$. Hence, applying Lemma 3.7 iteratively, so does the scheme $Y_{2}$ defined by $I_{P}^{k}$ for $\alpha-1 \geq k \geq 1$. Thus $T_{t, k}=A V_{X, t-k}(k)=0$ for $t \geq \alpha$. Thus all entries in region III are 0 , and we are done.

The next proposition shows that if a subscheme $X$ admits an unexpected hypersurface of degree $t$ vanishing with multiplicity $m$ at a general point, then $\left[\operatorname{gin}\left(I_{X}\right)\right]_{t}$ is not a lex-segment. This gives a criterion (this time algebraic), again based on a single piece of information (admittedly more difficult to verify), for the non-existence of any unexpected hypersurfaces.

Proposition 3.9. Let $X$ be a subscheme of $\mathbb{P}^{n}$. Assume there exists $j \geq 0$ such that $\left[\operatorname{gin}\left(I_{X}\right)\right]_{m+j}$ is a lex-segment and $\operatorname{adim}(X, m+j, m)>0$. Then $A V_{X, j}(m)=0$. In particular, if $\operatorname{gin}\left(I_{X}\right)$ is a lex-segment ideal, then $X$ does not admit any unexpected hypersurfaces.

Proof. Let $j \geq 0$ be an integer such that $X$ admits a hypersurface of degree $m+j$ vanishing with multiplicity at least $m$ at a general point $P$. Then by Lemma 3.1(i) we have $\operatorname{adim}(X, t, m)=\operatorname{dim}\left[\operatorname{gin}\left(I_{X}\right) \cap I_{Q}^{m}\right]_{t}>0$, so there is a monomial $x_{0}^{s} M \in \operatorname{gin}\left(I_{X}\right)$ where $M] \in I_{P}^{m}$ so has degree $\operatorname{deg} M=m+j-s \geq m$, but $j \geq s \geq 0$. Now, since $\left[\operatorname{gin}\left(I_{X}\right)\right]_{m+j}$ is a 
lex-segment, we have $x_{0}^{s} M \in \operatorname{gin}\left(I_{X}\right)$. In order to prove the statement, it is enough by Theorem 3.4 to show that $\operatorname{dim}\left[R /\left(\operatorname{gin}\left(I_{X}\right): x_{0}^{j+1}\right)\right]_{m-1}=0$. So, take any monomial $F \in R_{m-1}$. Then $x_{0}^{j+1} F \in[R]_{m+j}$ and $x_{0}^{j+1} F>_{\text {lex }} x_{0}^{s} M$. Thus, $x_{0}^{j+1} F \in \operatorname{gin}\left(I_{X}\right)$, so $F \in \operatorname{gin}\left(I_{X}\right): x_{0}^{j+1}$, hence $\left[R /\left(\operatorname{gin}\left(I_{X}\right): x_{0}^{j+1}\right)\right]_{m-1}=0$.

It is natural to ask if the converse of Proposition 3.9 is true, namely if it is true for a finite set of points $X$ that if $\operatorname{gin}\left(I_{X}\right)$ is not a lex-segment ideal then $X$ must admit some sort of unexpected hypersurface. Recalling that one must take with a grain of salt the generic initial ideal produced by a computer algebra program (is the change of variables "general enough?"), a counterexample is given in Example 3.10. It would be interesting to have a theoretical procedure to determine if the generic initial ideal of a finite set of points is a lex-segment ideal or not.

Example 3.10. Assume the characteristic of the field $K$ is 0 . Let $X_{n} \subseteq \mathbb{P}^{n}$ be the set of $\left(\begin{array}{c}n+2 \\ 2\end{array}\right)$ points obtained from the root system $A_{n+1}$ as described in [HMNT], section 3.1. Specifically, $X_{n}$ consists of the $\left(\begin{array}{c}n+1 \\ 2\end{array}\right)$ points having one entry equal to 1 , one equal to -1 and the rest 0 , together with the $n+1$ coordinate points.

The initial degree of $I_{X_{n}} \subseteq K\left[x_{0}, x_{1}, \ldots, x_{n}\right]$ is $\alpha\left(I_{X_{n}}\right)=3$ for $n \geq 2$, because the product of any 3 indeterminates vanishes at $X_{n}$ and $\left[I_{X_{n}}\right]_{2}=(0)$. To see that $X_{n}$ does not lie on a quadric we take $F:=\sum c_{a b} x_{a} x_{b} \in I_{X_{n}}$ and we show that $F=0$. Indeed, since $F$ vanishes at the coordinate points, $c_{a b}=0$ if $a=b$; also $c_{a b}=0$ for $a \neq b$ because $F$ vanishes at the point having no zero entries at the positions $a$ and $b$.

A computer calculation, by CoCoA $[\mathrm{ABR}]$, showed that $A V_{X_{n}, 0}(3)=0$ for $2 \leq n \leq 12$. Therefore, by Proposition $3.8, X_{n}$ does not admit any unexpected hypersurface of any sort, for $2 \leq n \leq 12$.

This result is consistent with [HMNT], where a computer search did not turn up any unexpected hypersurfaces for $X_{n}$ in the cases $2 \leq n \leq 6,2 \leq d \leq 6,2 \leq m \leq d$.

Furthermore, we checked with CoCoA that, for $4 \leq n \leq 12$, the ideal defining the set of points $X_{n} \subseteq \mathbb{P}^{n}$ has a generic initial ideal that is not a lex-segment ideal. In particular, in the cases $5 \leq n \leq 12$ we noticed that $\left[\operatorname{gin}\left(I_{X_{n}}\right)\right]_{3}$ fails to be a lex-segment because $x_{1} x_{n}^{2}$ fails to belong to gin $\left(I_{X_{n}}\right)$.

Interestingly, we have checked for $3 \leq n \leq 12$ that the set of points $Y_{n} \subseteq \mathbb{P}^{n}$, constructed from $X_{n}$ by replacing in the coordinates of its points all the " 1 " with " +1 ," admits an unexpected cone of degree 3 , and $x_{0} x_{n}^{2}$ fails to belong to $\operatorname{gin}\left(I_{Y_{n}}\right)$.

\section{On the SEQuence $A V_{X, 1}(m)$ When $X$ is an IRREDuCIBle ACM CURve in $\mathbb{P}^{3}$}

In this section we will assume that $K$ has characteristic zero. We have already seen in Theorem 3.4 that for a subscheme $X \subset \mathbb{P}^{n}$, the sequence $A V_{X, j}$ shifted to the left by 1 is an $O$-sequence. In this section we will abuse terminology and just say that the sequence is an $O$-sequence, often (but not always) suppressing the shift. Furthermore, if the positive part of the sequence $A V_{X, j}$ is finite, we will ignore the terms that are zero and just say that $A V_{X, j}$ is finite. In this section our focus is on the case $j=1$.

Recall that an SI-sequence is a finite, non-zero, symmetric $O$-sequence such that the first half is a differentiable $O$-sequence (i.e., also the first difference of the first half is an $O$-sequence). The significance of SI-sequences is that they characterize the $h$-vectors of arithmetically Gorenstein subschemes of projective space whose artinian reductions have the Weak Lefschetz Property. In codimension three they characterize the $h$-vectors of all 
arithmetically Gorenstein subschemes. Note that SI-sequences are automatically unimodal. See $[\mathrm{Hrm}]$ for properties of SI-sequences.

We have produced a great deal of experimental evidence for the following conjecture concerning SI-sequences. We will shortly prove part of it. We recall that an ACM scheme is always connected [E, Theorem 18.12], hence a smooth ACM scheme is irreducible.

Conjecture 4.1. Let $X \subset \mathbb{P}^{3}$ be a smooth $A C M$ curve not lying on a quadric surface. Then the sequence $A V_{X, 1}$ is an SI-sequence (shifted by 1 ). The last non-zero term in this sequence is $A V_{X, 1}(\operatorname{deg} X-5)$, so the SI-sequence ends in degree $\operatorname{deg} X-6$.

Remark 4.2. We point out two things. First, we have not yet seen a direct connection to Gorenstein algebras; we have only this numerical conjecture. It would be very interesting to tie Gorenstein algebras to the study of unexpected hypersurfaces in some way. Second, sometimes the SI-sequences that we obtain have codimension three and sometimes codimension four.

The case where $X$ does lie on a quadric surface is contained in Theorem 4.4. We first give examples to show that all of the other assumptions are needed in this conjecture.

Example 4.3. The symmetry of the sequence $A V_{X, 1}(m)$ requires all of the given assumptions. The following examples, which were run in either CoCoA [ABR] or Macaulay2 [GS], show that dropping a hypothesis can result in the AV sequence either not being finite, or, if finite, not being symmetric.

(a) ( $X$ satisfies all assumptions.) Assume that $X_{1}$ is a line in $\mathbb{P}^{3}$ and $X$ is obtained as the residual to $X_{1}$ by two general cubic surfaces containing $X_{1}$. Then $X$ is a smooth ACM curve of degree 8 and genus 7 , and the positive part of the sequence $A V_{X, 1}$ is $(1,2,1)$. This satisfies Conjecture 4.1 , and in particular it is symmetric with its last value being in degree $8-6=2$.

(b) ( $X$ is not $A C M$.) Assume that $X_{1}$ is the disjoint union of two lines in $\mathbb{P}^{3}$ and $X$ is linked to $X_{1}$ by a general choice of two cubic surfaces. Then $X$ is a smooth, non-ACM curve of degree 7 and genus 4 , and the positive part of the sequence $A V_{X, 1}$ is $(1,2)$, which is not symmetric.

(c) ( $X$ is not in $\mathbb{P}^{3}$.) Assume that $X$ is a smooth surface of degree 8 in $\mathbb{P}^{4}$ obtained from a plane by linking using two general hypersurfaces of degree 3. (The example in (a) is a hyperplane section of this one.) Then the positive part of the sequence $A V_{X, 1}$ is $(1,3,4,4, \ldots)$, which is not finite. (Note that its first difference is the sequence in (a).)

(d) ( $X$ is not equidimensional, but the curve part is ACM.) Assume that $X$ is the residual in $\mathbb{P}^{3}$ of a line inside the complete intersection of two cubics (as in (a)). Let $Y$ be the union of $X$ with a general point. Then the positive part of $A V_{Y, 1}$ is the sequence $(1,3,2)$, which is not symmetric.

(e) $(j=0$ instead of $j=1$.) Assume that $X$ is the curve in (a) but take $j=0$. Then the positive part of $A V_{X, 0}$ is the sequence $(1,4,8,11,13,14,14,14, \ldots)$, which is not finite.

(f) $(j=2$ instead of $j=1$.) Assume that $X$ is the curve in (a) but take $j=2$. Then $A V_{X, 2}$ is the sequence $(0,0, \ldots)$, and so is finite and vacuously symmetric, but does not end in the conjectured degree. However, linking the line $X_{1}$ from (a) using two surfaces of degree 4 gives a curve $X$ of degree 15 and genus 28, with the positive part of $A V_{X, 2}$ being the sequence $(1,2,2)$, which is not symmetric. 
(g) ( $X$ is zero-dimensional.) Assume that $X$ is the complete intersection in $\mathbb{P}^{3}$ of three general quartic surfaces. Then the positive part of the sequence $A V_{X, 1}$ is $(1,4,7,8,5)$ which is not symmetric.

(h) ( $X$ is $A C M$ but not irreducible, 1.) Let $Z \subset \mathbb{P}^{2}$ be the set of 9 points coming from the $B_{3}$ root system (see [DIV, Figure 2] and [HMNT]). Let $X$ be the cone over $Z$ with vertex at a general point $Q$. Then $X$ is an ACM union of lines (whose general hyperplane section admits an unexpected quartic curve), and the positive part of the sequence $A V_{X, 1}$ is $(1,3,4,4,4, \ldots)$, which is not finite.

(i) ( $X$ is $A C M$ but not irreducible, 2). Let $X$ be as in (h), except now let $Z \subset \mathbb{P}^{2}$ be a general set of 9 points. The Hilbert function of $Z$ (and hence $X$ ) is the same as it was in (h), but now one computes that the positive part of the sequence $A V_{X, 1}$ is $(1,3,3,3, \ldots)$, which is not finite. (Thus the AV sequence depends on geometry beyond the Hilbert function.)

(j) ( $X$ is $A C M$ and irreducible but not smooth). Let $Q$ be a general point and let $X$ be the complete intersection of two general quartic surfaces in $I_{Q}^{3}$. Then $X$ is ACM and we have verified that $X$ is irreducible (using Macaulay2), but the positive part of the sequence $A V_{X, 1}$ is $(1,4,8,12,15,16,15,12,8,4,2,2,2, \ldots)$, which is not finite. (Thus we need smoothness and not simply irreducibility in the statement of the conjecture.)

While we are not able to prove the full conjecture, we at least show unimodality and the differentiability of the increasing part, using only irreducibility of $X$ and not necessarily smoothness. What is missing is the finiteness, the symmetry and the degree of the last positive term in the sequence when $X$ is smooth. Notice that nothing about our argument fails for (i) or (j) in Example 4.3, so something more will be needed to prove the rest of the conjecture.

Theorem 4.4. Let $X \subset \mathbb{P}^{3}$ be an irreducible $A C M$ curve.

(a) If $X$ lies on a quadric surface, then for each $j \geq 1$ the sequence $A V_{X, j}(m)$ is zero.

(b) If $X$ does not lie on a quadric surface then the sequence $A V_{X, 1}(m)$ is non-zero and unimodal. Furthermore, the increasing part is a differentiable O-sequence.

Proof. (a) This follows by Corollary 3.5.

(b) We assume that

- $P \in \mathbb{P}^{3}$ is a general point with defining ideal $I_{P}$,

- $H$ is a general plane in $\mathbb{P}^{3}$ containing $P$,

- $L \in I_{P}$ is a linear form defining $H$,

- $Z=X \cap H$.

Note that $P$ may be taken to be a general point in $H$ with respect to the set $Z$. In addition to the notation introduced in Remark 2.12, recall from Notation 2.10 that

we will denote the scheme defined by $I_{P \mid H}^{m}$ by $m P$, a fat point in the plane, to distinguish it from the fat point scheme $P^{m}$ in $\mathbb{P}^{3}$ defined by $I_{P}^{m}$.

Notice that

$$
I_{X \cup P^{m+1}}: L=I_{X \cup P^{m}} \quad \text { and } \quad\left(I_{X \cup P^{m}}+(L)\right)^{s a t}=I_{Z \cup m P} .
$$

Since $Z=X \cap H$ is a general hyperplane section of $X$, and $X$ is irreducible, $Z$ is a set of points in linearly general position in $H$ (in this case meaning no three points of $Z$ are collinear). Hence by [CHMN] Corollary $6.8, Z$ does not admit any unexpected curves in the 
plane. Considering the exact sequence of sheaves

$$
0 \rightarrow \mathcal{I}_{Z \cup(m+1) P}(m+2) \rightarrow \mathcal{I}_{Z}(m+2) \stackrel{r_{m+2}}{\longrightarrow} \mathcal{O}_{(m+1) P}(m+2) \rightarrow 0
$$

from Remark 2.12 (where the ideal sheaves are on $H=\mathbb{P}^{2}$ ), the fact that $Z$ does not admit any unexpected curves means that $r_{m+2}$ has maximal rank on global sections. (For some values of $m$ it will be injective, and eventually it will be surjective.)

Now consider the commutative diagram of sheaves (the rows are exact since $P \notin X$ and the columns are exact since $X$ is irreducible):

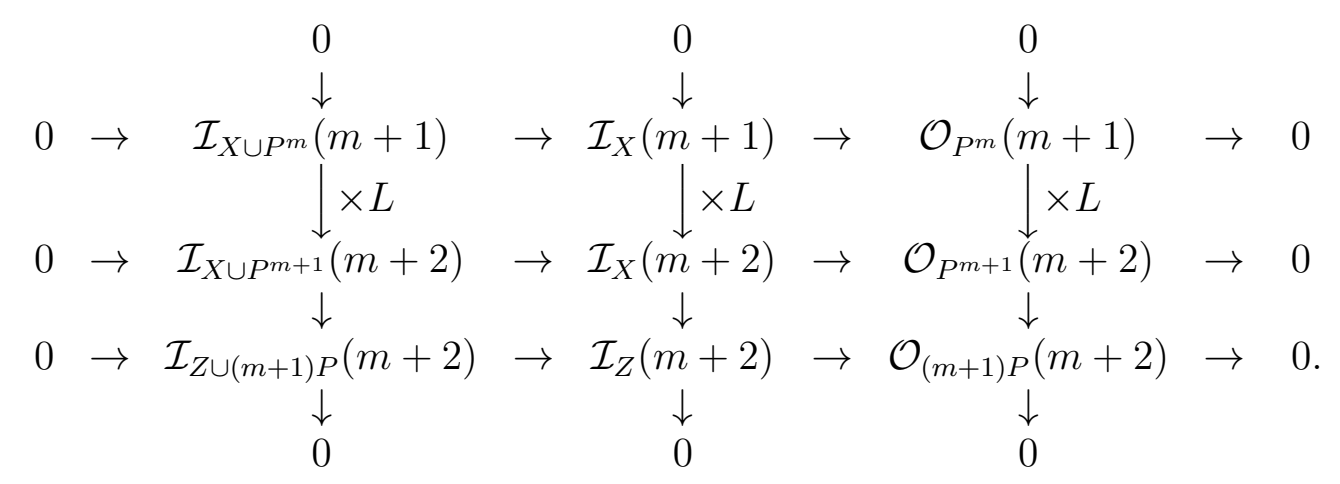

In cohomology we obtain

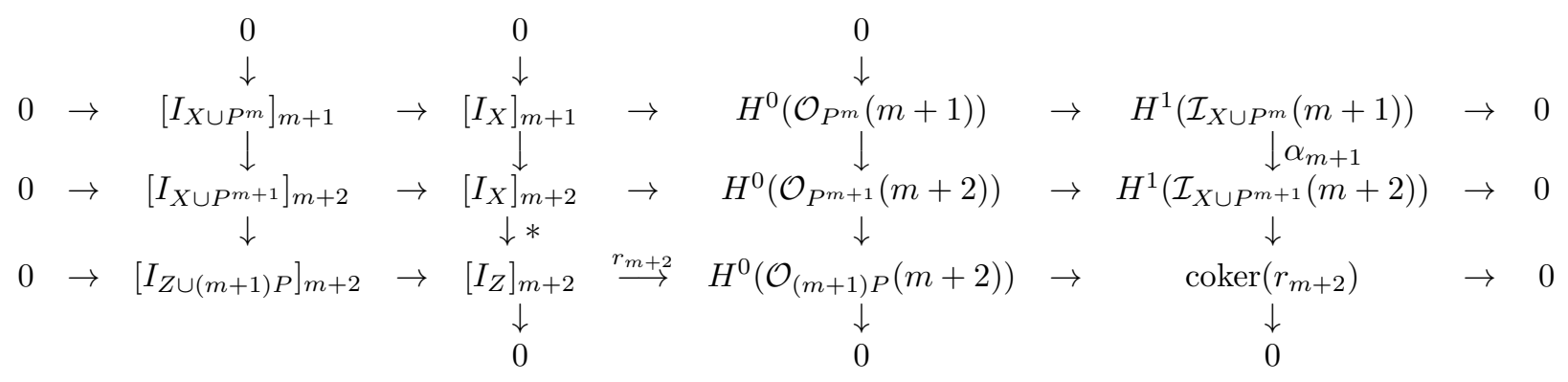

using the fact that $X$ is ACM for the first vertical surjection (marked by an asterisk).

To begin, we focus only on the first line of the above commutative diagram. For $m=0$ we obtain

$$
h^{1}\left(\mathcal{I}_{X \cup P^{0}}(1)\right)=h^{1}\left(\mathcal{I}_{X}(1)\right)=0
$$

since $X$ is ACM. For $m=1$ we obtain

$$
h^{1}\left(\mathcal{I}_{X \cup P}(2)\right)= \begin{cases}0 & \text { if } \operatorname{dim}\left[I_{X}\right]_{2} \geq 1 \\ 1 & \text { if } \operatorname{dim}\left[I_{X}\right]_{2}=0\end{cases}
$$

Since, by Remark 2.12 we have $h^{1}\left(\mathcal{I}_{X \cup P^{m}}(m+1)\right)=A V_{X, 1}(m)$, and we know the latter is an $O$-sequence shifted by one thanks to Theorem 3.4, this gives that the start of the shifted $O$-sequence (the value 1$)$ corresponds to $h^{1}\left(\mathcal{I}_{X \cup P}(2)\right)$. Thus the sequence is non-zero since $X$ does not lie on a quadric surface, so we have the first part of (b). (If we had merely assumed that $X$ is not degenerate, this also shows that the sequence is non-zero if and only if $X$ does not lie on a quadric surface.)

So for the rest of the proof we assume that $X$ does not lie on a quadric surface. Applying the Snake Lemma to the above commutative diagram, the fact that $r_{m+2}$ has maximal rank means that also $\alpha_{m+1}$ has maximal rank (in the right-hand column of the diagram). Then applying Remark 2.12 as $m$ varies, since $r_{m+2}$ is initially injective and then surjective, the same is true for the map $\alpha_{m+1}$. This shows that the sequence $A V_{X, 1}(m)$ is unimodal. 
Finally, we argue that the increasing part of the sequence $\left\{h^{1}\left(\mathcal{I}_{X \cup P^{m}}(m+1)\right)\right\}$ is a differentiable $O$-sequence (shifted by 2 ).

Thanks to Theorem 3.4 and Remark 2.12, we know that the sequence $\left\{h^{1}\left(\mathcal{I}_{X \cup P^{m}}(m+1)\right)\right\}$ is an $O$-sequence, and we have just seen that the map $\alpha_{m+1}$ is injective as long as $\operatorname{coker}\left(r_{m+2}\right)$ is non-zero. So we have only to show that $\operatorname{dim}\left(\operatorname{coker}\left(r_{m+2}\right)\right)$ is an $O$-sequence (shifted by 2). But because $r_{m+2}$ has maximal rank, when coker $\left(r_{m+2}\right)$ is non-zero we have from the exactness of the bottom row of the above diagram that

$$
\begin{aligned}
\operatorname{dim}\left[\operatorname{coker}\left(r_{m+2}\right)\right]_{m+2} & =\left(\begin{array}{c}
(m+1)-1+2 \\
2
\end{array}\right)-\operatorname{dim}\left[I_{Z}\right]_{m+2} \\
& =h_{R / I_{Z}}(m+2)-[2(m+2)+1]
\end{aligned}
$$

as long as this number is positive.

So for $t \geq 2$ we want to show that

$$
k_{t-2}:=h_{R / I_{Z}}(t)-(2 t+1)
$$

is an $O$-sequence as long as it is positive. Let $s=t-2$, so we want to show that $k_{s}$ is an $O$-sequence for $s \geq 0$. Since $X$ does not lie on a quadric surface, $Z$ does not lie on a conic (since $X$ is ACM). Thus $k_{0}=\operatorname{dim}\left[\operatorname{coker}\left(r_{2}\right)\right]_{2}=6-5=1$.

Now assume that $t \geq 3$, so $s \geq 1$. As long as $h_{R / I_{Z}}(t)=\left(\begin{array}{c}t+2 \\ 2\end{array}\right)$ (i.e., before $I_{Z}$ begins) this difference is $k_{s}=\left(\begin{array}{c}s+2 \\ 2\end{array}\right)$, which is an $O$-sequence. So assume $h_{R / I_{Z}}(t)<\left(\begin{array}{c}t+2 \\ 2\end{array}\right)$. Consider the $t$-binomial expansion of $h_{R / I_{Z}}(t)$. Since

$$
2 t+1<h_{R / I_{Z}}(t)<\left(\begin{array}{c}
t+2 \\
2
\end{array}\right)
$$

we have

$$
h_{R / I_{Z}}(t)=\left(\begin{array}{c}
t+1 \\
t
\end{array}\right)+\left(\begin{array}{c}
t \\
t-1
\end{array}\right)+(\text { terms in degrees } \leq t-2)
$$

and

$$
(2 t+1)=\left(\begin{array}{c}
t+1 \\
t
\end{array}\right)+\left(\begin{array}{c}
t \\
t-1
\end{array}\right)
$$

Hence $k_{s}=h_{R / I_{Z}}(t)-(2 t+1)$ has an $s$-binomial expansion coming directly from the $t$ binomial expansion of $h_{R / I_{Z}}(t)$, by removing the first two binomial coefficients. But $h_{R / I_{Z}}$ is an $O$-sequence, so it obeys Macaulay's bound (Theorem 2.2). Thus $k_{s}$ does as well, and so is an $O$-sequence.

\section{Codimension 2 COmplete intersections in $\mathbb{P}^{n}$}

In this section we apply results of Section 3 to the case of complete intersections of codimension 2 in $\mathbb{P}^{n}$. In particular, as introduced in Remark 3.2, we get partial information on the actual dimension for a complete intersection of codimension 2 in a certain degree by using the theory of partial elimination ideals. (See [Gr] for background on partial elimination ideals and for Sylvester matrices, which appear in the proof of Proposition 5.1.) Complete intersections of codimension 2 have been investigated in several papers. Indeed, the following result can be deduced from Proposition 6.8 in [Gr] and Corollary 3.9 in [CoS]. We include here a proof for completeness of the exposition and to show explicitly how the partial elimination theory affects the existence of unexpected hypersurfaces. 
Recall that $R=K\left[x_{0}, x_{1}, \ldots, x_{n}\right]$ denotes a standard graded polynomial ring and the monomials of $R$ are ordered by $>_{l e x}$, the lexicographic monomial order which satisfies $x_{0}>$ $x_{1}>\cdots>x_{n}$.

Proposition 5.1. Let $C \subseteq \mathbb{P}^{n}$ be a codimension 2 complete intersection. Assume $C$ is defined by two, sufficiently general, forms of degree $a, b$ respectively. Say $a \leq b$. Set, for any integer $0 \leq j<a$,

$$
\begin{aligned}
t & :=(a-j)(b-j)+j \text { and } \\
m & :=(a-j)(b-j) .
\end{aligned}
$$

Then

$$
\operatorname{adim}(X, t, m)>0 .
$$

Proof. After a general change of coordinates, say

$$
\begin{aligned}
& F=f_{a}+f_{a-1} x_{0}+f_{a-2} x_{0}^{2}+\cdots+f_{1} x_{0}^{a-1}+f_{0} x_{0}^{a} \\
& G=g_{b}+g_{b-1} x_{0}+g_{b-1} x_{0}^{2}+\cdots+g_{1} x_{0}^{b-1}+g_{0} x_{0}^{b}
\end{aligned}
$$

and define $J:=(F, G)$ to be the ideal generated by $F$ and $G$. Let $M$ be the following matrix of size $(a+b-2 j) \times(a+b-2 j)$ :

$$
M:=\left(\begin{array}{cccccccc}
f_{a-j} & f_{a-j-1} & \cdots & f_{1} & f_{0} & 0 & \cdots & 0 \\
f_{a-j+1} & f_{a-j} & \cdots & f_{2} & f_{1} & f_{0} & \cdots & 0 \\
\vdots & \vdots & \ddots & & & & & \\
f_{a} & f_{a-1} & \cdots & & & & & \\
0 & f_{a} & & & & & & \\
\vdots & \vdots & & & & & \\
0 & 0 & \cdots & & & \cdots & f_{0} \\
g_{b-j} & g_{b-j-1} & \cdots & & & \cdots & 0 \\
g_{b-j+1} & g_{b-j} & \cdots & & & \cdots & 0 \\
\vdots & \vdots & \ddots & & & & \\
g_{b} & g_{b-1} & \cdots & & & & \\
0 & g_{b} & \cdots & & & & \\
\vdots & \vdots & & & & & \\
0 & 0 & \cdots & & & \cdots & g_{0}
\end{array}\right) .
$$

(Note that the determinant of the matrix $M$ is one of the minors of the Sylvester matrix $\operatorname{Syl}\left(F, G, x_{0}\right)$ of $F$ and $G$, and these minors belong to the partial elimination ideal $K_{j}(J)$. See in particular Corollary 3.9 in $[\mathrm{CoS}]$ and Proposition 6.9 and the following Remark in [Gr].) One can check that $\operatorname{det}(M)$ is homogeneous and it has degree $m$. Let $M_{i}$ denote the cofactor of $M$ corresponding to $(-1)^{i+1}$ times the determinant of the matrix that results from deleting the $i$-th row and the first column of $M$. Consider the following $a+b-2 j$ 
polynomials

$$
\begin{aligned}
& F=f_{a}+f_{a-1} x_{0}+\cdots+f_{a-j} x_{0}^{j}+f_{a-j-1} x_{0}^{j+1}+\cdots \\
& x_{0} F=f_{a} x_{0}+\cdots+f_{a-j+1} x_{0}^{j}+f_{a-j} x_{0}^{j+1}+\cdots \\
& \text { : } \\
& x_{0}^{j-1} F=f_{a} x_{0}^{j}+f_{a-1} x_{0}^{j+1}+\cdots \\
& x_{0}^{b-j-1} F=\cdots \\
& G=g_{b}+g_{b-1} x_{0}+\cdots+g_{b-j} x_{0}^{j}+g_{b-j-1} x_{0}^{j+1}+\cdots \\
& x_{0} G=g_{b} x_{0}+\cdots+g_{b-j+1} x_{0}^{j}+g_{b-j} x_{0}^{j+1}+\cdots \\
& \vdots \\
& x_{0}^{a-j-1} G=\cdots
\end{aligned}
$$

Multiplying the above $a+b-2 j$ polynomials respectively by $M_{1}, M_{2}, \ldots, M_{a+b-2 j}$ and taking the sum of them, we get

$$
\begin{aligned}
T:=\left(\sum_{i=1}^{b-j} M_{i} x_{0}^{i-1}\right) F & +\left(\sum_{i=1}^{a-j} M_{b-j+i} x_{0}^{i-1}\right) G= \\
& =f_{a} M_{1}+g_{b} M_{b-j+1}+x_{0}(\cdots)+\cdots+x_{0}^{j}(\cdots)+x_{0}^{j+1}(\cdots)+\cdots
\end{aligned}
$$

Note that in the form $T \in K\left[x_{1}, \ldots x_{n}\right]\left[x_{0}\right]$ the coefficient of $x_{0}^{j}$ is $\operatorname{det}(M)$. Also, note that all the coefficients of the powers of $x_{0}$ greater than $j$ are zero; for instance the coefficient of $x_{0}^{j+1}$ is the sum of the entries of the second column in $M$ multiplied by the cofactors of the entries in the first column. Of course $T$ belongs to the ideal generated by $F$ and $G$, therefore, since we performed a general change of variables, its leading term belongs to in $(J)=\operatorname{gin}\left(I_{C}\right)$. As noted above, the form $T$ can be written as $T=T_{m+j}+x_{0}\left(T_{m+j-1}\right)+\cdots+x_{0}^{j}\left(T_{m}\right)$ where $T_{i} \in K\left[x_{1}, \ldots x_{n}\right]_{i}$ and $T_{m}=\operatorname{det}(M)$, thus in $(T)=x_{0}^{j} \cdot \operatorname{in}(\operatorname{det}(M)) \in \operatorname{gin}\left(I_{C}\right)$. Since in $(T)$ vanishes with multiplicity $m$ at the point $Q:=(1,0, \ldots, 0)$, by Lemma 3.1 , we are done.

Remark 5.2. As a consequence of Proposition 5.1, a general codimension 2 complete intesection $C \subseteq \mathbb{P}^{n}$ admits an unexpected hypersurface of degree $t$ for multiplicity $m$ whenever $\operatorname{edim}(C, t, m)=0$, i.e. $\operatorname{vdim}(C, t, m) \leq 0$. In order to compute edim $(C, t, m)$, where $C$ is a complete intersection of type $(a, b)$ in $\mathbb{P}^{n}$, take the short exact sequence

$$
0 \rightarrow R(-a-b) \rightarrow R(-a) \oplus R(-b) \rightarrow I_{C} \rightarrow 0 .
$$

So, computing the dimension of the graded pieces of degree $t$, we get

$$
\operatorname{dim}\left[I_{C}\right]_{t}=\left(\begin{array}{c}
t-a+n \\
n
\end{array}\right)+\left(\begin{array}{c}
t-b+n \\
n
\end{array}\right)-\left(\begin{array}{c}
t-a-b+n \\
n
\end{array}\right) .
$$

Therefore, we have

$$
\operatorname{edim}(C, t, m)=\max \left\{\operatorname{dim}\left[I_{C}\right]_{t}-\left(\begin{array}{c}
m-1+n \\
n
\end{array}\right), 0\right\}
$$

Remark 5.3. The case $j=0$ is covered by [HMNT, Proposition 2.4]. The cone with vertex $P$ over a codimension 2 complete intesection $C \subseteq \mathbb{P}^{n}$ of type $(a, b)$ is an unexpected hypersurface for $C$ of degree $a b$ and multiplicity $a b$ at $P$. 
If $j \geq 1$ it is enough to show that $\operatorname{vdim}(C,(a-j)(b-j)+j,(a-j)(b-j)) \leq 0$ to ensure the existence of an unexpected hypersurface. In the next proposition we deal with the case $j=1$ in $\mathbb{P}^{3}$. Theorem 4.4 shows that a general complete intersection of type $(2, b)$ never admits an unexpected hypersurface with $j=1$. The following shows that when $a>2$ we do obtain unexpected hypersurfaces.

Proposition 5.4. Let $C \subseteq \mathbb{P}^{3}$ be a general codimension 2 complete intersection defined by two forms of degree $a, b$ respectively. Say $a \leq b$. Set $t:=(a-1)(b-1)+1$ and $m:=(a-1)(b-1)$. If $a>2$ then $C$ admits an unexpected hypersurface of degree $t$ for multiplicity $m$.

Proof. From Remark 5.2 we have

$$
\operatorname{vdim}(C, t, m)=\left(\begin{array}{c}
t-a+3 \\
3
\end{array}\right)+\left(\begin{array}{c}
t-b+3 \\
3
\end{array}\right)-\left(\begin{array}{c}
t-a-b+3 \\
3
\end{array}\right)-\left(\begin{array}{c}
t-2+3 \\
3
\end{array}\right) .
$$

If $a=b=3$ then $t=5$ and

$$
\operatorname{vdim}(C, t, m)=\left(\begin{array}{l}
5 \\
3
\end{array}\right)+\left(\begin{array}{l}
5 \\
3
\end{array}\right)-\left(\begin{array}{l}
6 \\
3
\end{array}\right) \leq 0 .
$$

A similar computation follows if $a=3$ and $b=4$. Note that, in this case $t+3-a-b=3$. Since the integer $t+3-a-b$ increases with $a$ and $b$, in particular if $(a, b)>(3,4)$ we have $t+3-a-b>3$, so the binomial coefficient $\left(\begin{array}{c}t+3-a-b \\ 3\end{array}\right)$ is not zero. Then, assuming $(a, b)>(3,4)$, after a standard computation we get

$$
\begin{aligned}
\operatorname{vdim}(C, t, m) & =-\frac{1}{2} a^{2} b-\frac{1}{2} a b^{2}+a^{2}+b^{2}+4 a b-6 a-6 b+9= \\
& =-\frac{1}{2}(a-2)(b-2)(a+b-4)+1 \leq 0 .
\end{aligned}
$$

Thus $\operatorname{vim}(C, t, m) \leq 0$.

Remark 5.5. Question 2.11 of [HMNT] asks the following: Let $Z$ be a non-degenerate set of points in linear general position in $\mathbb{P}^{n}, n \geq 3$. Is it true that there does not exist an unexpected hypersurface of any degree $t$ and multiplicity $m=t-1$ at a general point? (All other possible combinations of $(n, t, m)$ are settled.)

The work in this section allows us to give a negative answer to this question. Indeed, let $X$ be a general complete intersection of type $(3,3)$ in $\mathbb{P}^{n}(n \geq 3)$. Then let $t=(3-1)(3-1)+1=$ 5 and $m=t-1=4$. From Remark 5.2 we have

$$
\operatorname{vdim}(X, t, m)=\left(\begin{array}{c}
n+2 \\
n
\end{array}\right)+\left(\begin{array}{c}
n+2 \\
n
\end{array}\right)-\left(\begin{array}{c}
n-1 \\
n
\end{array}\right)-\left(\begin{array}{c}
n+3 \\
n
\end{array}\right) \leq 0
$$

for all $n \geq 3$. On the other hand, Proposition 5.1 gives (with $a=b=3$ and $j=1$ ) that $X$ does lie on a hypersurface of degree 5 and multiplicity 4 at a general point, so this hypersurface is unexpected. Now take a set $Z$ consisting of sufficiently many general points on $X$, so that $\left[I_{X}\right]_{5}=\left[I_{Z}\right]_{5}$. Then $Z$ is in linear general position since $X$ is irreducible, and so $Z$ admits an unexpected hypersurface and gives a negative answer to the question.

We believe that the natural extension of Proposition 5.4 to $\mathbb{P}^{n}$ is also true, but we do not have a proof. 
Remark 5.6. One could make the following objection to Remark 5.5 as an answer to Question 2.11 of [HMNT]. That is that the degree 5 component of $I_{Z}$ is the same as the degree 5 component of $I_{X}$, so the base locus of $\left[I_{Z}\right]_{5}$ is $X$ and the geometry is really only about $X$ and not about $Z$.

To respond to this, we make the following tweak. Take $n=4$. We choose the same $X$ as above (now a surface in $\mathbb{P}^{4}$ ), and we still take $t=5, m=4$. One checks that the Hilbert function of $R / I_{X}$ is

$$
1,5,15,33,60,96,141 \ldots
$$

and we still have

$$
\operatorname{vdim}(X, t, m)=\left(\begin{array}{c}
4+2 \\
4
\end{array}\right)+\left(\begin{array}{c}
4+2 \\
4
\end{array}\right)-\left(\begin{array}{c}
4-1 \\
4
\end{array}\right)-\left(\begin{array}{c}
4+3 \\
4
\end{array}\right)=-5 .
$$

But $X$ contains a set $Z$ of 225 points giving a $(3,3,5,5)$ complete intersection, hence

$$
\operatorname{dim}\left[I_{Z}\right]_{5}=32=\operatorname{dim}\left[I_{X}\right]_{5}+2
$$

so $\operatorname{vdim}(Z, t, m)=-3$, hence all quintic hypersurfaces containing $Z$ with a general point of multiplicity 4 are unexpected, and there is certainly at least one (this being the one that contains $X$ ). There still remains an objection: computations show in this case that $X$ and $Z$ both have a unique unexpected quintic for multiplicity 4 , so the one for $Z$ is the same one that we already got for $X$. Nevertheless, what we have gained is that the component of the ideal for $Z$ in degree 5 is no longer the same as that of $X$, and indeed the base locus now is finite while the base locus in the original example was $X$ itself.

\section{Cones, unmixed CuRves And unions with finite sets of Points in $\mathbb{P}^{3}$}

In this section, for the most part we restrict our attention to the case of subvarieties of $\mathbb{P}^{3}$. We recall that

$$
A V_{X, 0}(t)=\operatorname{adim}(X, t, t)-\operatorname{vdim}(X, t, t) .
$$

We will determine the $A V_{Z, 0}$ sequence for the cases where $Z=C$ is an equidimensional curve in $\mathbb{P}^{3}$ and where $Z=C \cup X$ is the union of a curve $C$ and a finite set of points $X$. In particular, since $j=0$, we are focusing on the case of unexpected cones. In both cases the geometric information on $C$ provides a description of the persistence of unexpected hypersurfaces. We recall the following result, which reflects the persistence of unexpectedness for cones for a non-degenerate curve in $\mathbb{P}^{3}$.

Theorem 6.1 ([HMNT] Corollary 2.12). Let $C \subset \mathbb{P}^{3}$ be a reduced, equidimensional, nondegenerate curve of degree $d \geq 2$ ( $C$ may be reducible, singular, and/or disconnected). Let $P \in \mathbb{P}^{3}$ be a general point. Let $k \geq d$ be a positive integer. Then $C$ admits an unexpected hypersurface of degree $k$ with multiplicity $k$ at $P$. When $k=d$, this hypersurface is unique.

Our next result not only reproduces the persistence given by the result of Theorem 6.1 but also gives a measure of the unexpectedness in each degree.

Theorem 6.2. Let $C \subset \mathbb{P}^{3}$ be a reduced, equidimensional curve of degree e and arithmetic genus $g$. Then

$$
A V_{C, 0}(t)=\left(\begin{array}{c}
e-1 \\
2
\end{array}\right)-g
$$

for all $t \geq e$. Moreover, $A V_{C, 0}(e)=0$ if and only if $A V_{C, 0}(t)=0$ for $t \geq e$ if and only if $C$ lies in a plane. 
Proof. Let $P$ be a general point. We are considering hypersurfaces of degree $t$ with multiplicity $t$ at $P$, which are cones with vertex $P$ that contain $C$. Since the projection of $C$ from $P$ is contained in the hyperplane section of such a cone, we must have $t \geq e$. We first compute the virtual dimension of $I_{C \cup P^{t}}$ :

$$
\begin{aligned}
\operatorname{dim}\left[I_{C}\right]_{t}-\left(\begin{array}{c}
t+2 \\
3
\end{array}\right) & =\left(\begin{array}{c}
t+3 \\
3
\end{array}\right)-h_{C}(t)-\left(\begin{array}{c}
t+2 \\
3
\end{array}\right)= \\
& =\left(\begin{array}{c}
+2 \\
2
\end{array}\right)-[t e-g+1] .
\end{aligned}
$$

(The fact that for $t \geq e$ we have $h_{C}(t)=t e-g+1$ follows from the main result of [GLP].) On the other hand, if $S$ is such a cone of degree $t$ and if $Q$ is a point of $C$, then the line joining $P$ and $Q$ meets $S$ with multiplicity at least $t+1$, so it must lie on $S$. That is, $S$ contains as a component the cone over $C$ with vertex $P$. Then the actual dimension of $I_{C \cup P^{t}}$ is the (vector space) dimension of the linear system of plane curves of degree $t-e$, i.e. it is $\left(\begin{array}{c}t-e+2 \\ 2\end{array}\right)$. Then we obtain

$$
A V_{C, 0}(t)=\left(\begin{array}{c}
e-1 \\
2
\end{array}\right)-g
$$

after a simple calculation. The last part follows since $g=\left(\begin{array}{c}e-1 \\ 2\end{array}\right)$ if and only if $C$ is a plane curve, [HMNT, Proposition 2.1, Claim 1].

Even if your interest is for the case when $Z$ is a finite set of points (which we consider in the next section), situations involving curves (such as we are looking at in this section) sometimes force themselves into the picture in subtle ways, as the next example shows.

Example 6.3. Let $X_{1}, X_{2} \subset \mathbb{P}^{3}$ be finite sets of points with $h$-vectors, respectively,

$$
(1,3,6,5,3,3,2) \quad \text { and } \quad(1,3,6,6,3,3,2) \text {. }
$$

In both cases, the two 3's constitute maximal growth, viewing the $h$-vector as a Hilbert function, and force "many" of the points to lie on a curve of degree 3 [BGM]. For the sake of this example, let us assume that in both cases this curve is a twisted cubic, that it contains 18 points of each of $X_{1}$ and $X_{2}$ (with $h$-vector $(1,3,3,3,3,3,2)$ in both cases), and that the remaining 5 points of $X_{1}$ and the remaining 6 points of $X_{2}$ are chosen generically. Then in the first case there is a unique unexpected cone of degree 5 with vertex at a general point, while in the second case there is no unexpected cone of degree 5. We omit details here since we will study this kind of situation in the next section (see especially Example 7.4).

Our next result can again be viewed as a measure of unexpectedness for cones in each degree, and a statement about the persistence of unexpected cones.

Theorem 6.4. Let $X \subset \mathbb{P}^{3}$ be a finite set of points. Let $C$ be a reduced, equidimensional curve of degree $e$ and arithmetic genus $g$. Assume that $X$ is disjoint from $C$. Let $t$ be the smallest integer such that

(i) $|X|<\left(\begin{array}{c}t+2 \\ 2\end{array}\right)$, and

(ii) $X$ imposes independent conditions on forms of degree $t$.

Then

$$
A V_{X \cup C, 0}(t+e)=A V_{X, 0}(t)+\left[\left(\begin{array}{c}
e-1 \\
2
\end{array}\right)-g\right] .
$$

Proof. Let $P$ be a general point. Let $S_{P}$ be the cone over $C$ with vertex $P$; we know $\operatorname{deg} S_{P}=e, S_{P}$ is reduced, and $S_{P}$ has multiplicity $e$ at $P$. Let $T_{P}$ be a surface (unmixed) of degree $t+e$ containing $X \cup C$ and having multiplicity $t+e$ at $P$. ( $T_{P}$ is a cone over a 
suitable plane curve.) Note that $S_{P}$ is a component of $T_{P}$, and note that since $P$ is general, no point of $X$ lies on $S_{P}$. Write $U_{P}$ for the residual to $S_{P}$ in $T_{P}$. Note that $\operatorname{deg} U_{P}=t, U_{P}$ has multiplicity $t$ at $P$ and $U_{P}$ contains $X$. We also know that $\operatorname{adim}(C, e, e)=1$ (Theorem 6.1). These observations imply

$$
\operatorname{dim}\left[I_{X} \cap I_{P}^{t}\right]_{t}=\operatorname{dim}\left[I_{X \cup C} \cap I_{P}^{t+e}\right]_{t+e} .
$$

We also remark that since $X$ imposes independent conditions on forms of degree $t$ and $C$ imposes independent conditions on forms of degree $e$ (by [GLP]), we can conclude

$$
X \text { also imposes independent conditions on }\left[I_{C}\right]_{t+e} \text {. }
$$

By definition we have

$$
A V_{X, 0}(t)=\operatorname{dim}\left[I_{X} \cap I_{P}^{t}\right]_{t}-\left[\operatorname{dim}\left[I_{X}\right]_{t}-\left(\begin{array}{c}
t+2 \\
3
\end{array}\right)\right] .
$$

Now we compute (using the observation about independent conditions)

$$
\begin{aligned}
A V_{X \cup C, 0}(t+e) & =\operatorname{dim}\left[I_{X \cup C} \cap I_{P}^{t+e}\right]_{t+e}-\left[\operatorname{dim}\left[I_{X \cup C}\right]_{t+e}-\left(\begin{array}{c}
t+e+2 \\
3
\end{array}\right)\right] \\
& =\operatorname{dim}\left[I_{X} \cap I_{P}^{t}\right]_{t}-\left[\operatorname{dim}\left[I_{X \cup C}\right]_{t+e}-\left(\begin{array}{c}
t+e+2 \\
3
\end{array}\right)\right] \\
& =A V_{X, 0}(t)+\left[\operatorname{dim}\left[I_{X}\right]_{t}-\left(\begin{array}{c}
t+2 \\
3
\end{array}\right)\right]-\left[\operatorname{dim}\left[I_{X \cup C}\right]_{t+e}-\left(\begin{array}{c}
t+e+2 \\
3
\end{array}\right)\right] \\
& =A V_{X, 0}(t)+\left[\left(\begin{array}{c}
t+3 \\
3
\end{array}\right)-|X|-\left(\begin{array}{c}
t+2 \\
3
\end{array}\right)\right]-\left(\operatorname{dim}\left[I_{C}\right]_{t+e}-|X|\right)+\left(\begin{array}{c}
t+e+2 \\
3
\end{array}\right) \\
& =A V_{X, 0}(t)+\left(\begin{array}{c}
t+2 \\
2
\end{array}\right)-\operatorname{dim}\left[I_{C}\right]_{t+e}+\left(\begin{array}{c}
t+e+2 \\
3
\end{array}\right) \\
& =A V_{X, 0}(t)+\left(\begin{array}{c}
t+2 \\
2
\end{array}\right)-\left[\left(\begin{array}{c}
t+e+3 \\
3
\end{array}\right)-(e(t+e)-g+1)\right]+\left(\begin{array}{c}
t+e+2 \\
3
\end{array}\right) \\
& =A V_{X, 0}(t)+\left(\begin{array}{c}
e-1 \\
2
\end{array}\right)-g
\end{aligned}
$$

(the fourth line uses (6.1) and the last line comes after a routine calculation).

Corollary 6.5. Let $X \subset \mathbb{P}^{3}$ be a finite set of points. Let $C$ be a reduced plane curve in $\mathbb{P}^{3}$ of degree $d$ disjoint from $X$. Then $X$ has an unexpected cone of degree $t$ if and only if $X \cup C$ has an unexpected cone of degree $t+d$. Furthermore, $A V_{X, 0}(t)=A V_{X \cup C, 0}(t+d)$ for all $t$.

Proof. The arithmetic genus of a plane curve of degree $e$ is $\left(\begin{array}{c}e-1 \\ 2\end{array}\right)$.

\section{Finite Sets OF POINTS In $\mathbb{P}^{3}$}

In this section we assume that $K$ has characteristic zero. One of the original motivations for this paper was to determine if there are any Hilbert functions for non-degenerate sets of points that force the existence of unexpected hypersurfaces of some sort. (A consequence of Corollary 3.5 is that given a finite $O$-sequence $\left(1, a_{1}, \ldots, a_{r}\right)$, one can trivially find a set of points in some projective space, with this $h$-vector, that does not admit any unexpected 
hypersurfaces. One simply produces a set $X$ in $\mathbb{P}^{n}$ for $n>a_{1}$ having this $h$-vector. Then $X$ is degenerate, hence admits no unexpected hypersurfaces. Thus it is more interesting to consider non-degenerate sets.)

It now seems plausible that in a strict sense there are no such Hilbert functions. Indeed, we make the following conjecture (but see Theorem 7.8 and Corollary 7.10, which show that such Hilbert functions do arise when combined with a little geometric information).

Conjecture 7.1. For every possible $h$-vector $\left(1, n, a_{2}, \ldots, a_{r}\right)$ for a non-degenerate, finite set of points in $\mathbb{P}^{n}$, there is a set of points $X$ with that h-vector such that $X$ does not admit any unexpected hypersurfaces of any degree and multiplicity.

In trying to prove Conjecture 7.1 we made the following observations. Recall that a distraction is a construction that converts, in particular, an artinian monomial ideal in $K\left[x_{0}, \ldots, x_{n-1}\right]$ to the ideal of a reduced set of points in $\mathbb{P}^{n}$. It was introduced in [Hart]. See also $[\mathrm{MN}]$ for related constructions and results.

One way of constructing a reduced set of points with a given $h$-vector is to start with the artinian lex-segment ideal with Hilbert function $h$ and perform a distraction to produce a set of points $X$. Experimentally, it seems that very often $\operatorname{gin}\left(I_{X}\right)$ is a lex-segment ideal. If this were always the case, we would be done by Corollary 7.2 :

Corollary 7.2. Let $h=\left(1, n, a_{2}, \ldots, a_{r}\right)$ be a finite $O$-sequence. Let $X \subset \mathbb{P}^{n}$ be a set of points with this h-vector. If $\operatorname{gin}\left(I_{X}\right)$ is a lex-segment ideal in $R=K\left[x_{0}, x_{1}, \ldots, x_{n}\right]$, then $X$ does not admit any unexpected hypersurfaces, for any degree and multiplicity.

Proof. This follows immediately from Proposition 3.9.

Unfortunately, we have verified that the $h$-vector $(1,3,6,10,5,5,2)$ results in a set of points for which $\operatorname{gin}\left(I_{X}\right)$ is not a lex-segment ideal, and in fact one can check on CoCoA that it admits an unexpected hypersurface of degree 4 with multiplicity 3 . However, we were able to confirm that the union in $\mathbb{P}^{3}$ of 22 general points on a plane curve of degree 5 and 10 general points in $\mathbb{P}^{3}$ results in a set of points with the desired $h$-vector, which does not admit any unexpected hypersurfaces, so the conjecture is still true for this $h$-vector even though the distraction does not produce the desired set of points. This should be contrasted with the end of Example 7.4; in this case we verified that the distraction does produce a set of points whose gin is a lex-segment. Thus the conjecture remains open.

We recall a result from $[\mathrm{BGM}]$, modified to fit our context. For a set of points $X$ we denote by $\left\langle\left[I_{X}\right]_{\leq d}\right\rangle$ the ideal generated by the polynomials in $I_{X}$ of degree $\leq d$. Recall also that for a subscheme $Y$ of $\mathbb{P}^{n}$ we denote by $h_{Y}(t)$ its Hilbert function.

Proposition 7.3 ([BGM] Theorem 3.6). Let $X \subset \mathbb{P}^{3}$ be a reduced, finite set of points with $h$-vector

where $d \leq k+1$. Then

$$
\left(1,3, a_{2}, a_{3}, \ldots, a_{k}, d, d, a_{k+3}, \ldots, a_{r}\right)
$$

(a) $\left\langle\left[I_{X}\right]_{\leq k+1}\right\rangle$ is the saturated ideal of a reduced curve, $V$, of degree $d$ (not necessarily unmixed). Also, $I_{X}$ has no minimal generators in degree $k+2$, so $\left\langle\left[I_{X}\right]_{\leq k+1}\right\rangle=$ $\left\langle\left[I_{X}\right]_{\leq k+2}\right\rangle$.

(b) Let $C$ be the unmixed, one-dimensional part of $V$. Let $X_{1}$ be the subset of $X$ on $C$ and let $X_{2}$ be the subset of $X$ not on $C$; note $X=X_{1} \cup X_{2}$. Then $\left\langle\left[I_{X_{1}}\right]_{\leq k+1}\right\rangle=I_{C}$, and $V=C \cup X_{2}$. 
(c) $h_{X_{1}}(t)=h_{X}(t)-\left|X_{2}\right|$ for all $t \geq k$.

(d)

$$
\Delta h_{X_{1}}(t)= \begin{cases}\Delta h_{C}(t) & \text { for } t \leq k+2 \\ \Delta h_{X}(t) & \text { for } t \geq k+1\end{cases}
$$

From now on we focus on the special case $j=0$, i.e. when the degree of the unexpected hypersurface is equal to the multiplicity at the general point. The following example shows that knowing the $h$-vector of a finite set of points, and taking into account the fact that the base locus of some component of $I_{X}$ contains a curve, is not enough to ensure that $X$ has an unexpected surface. We generally have to know something more about the curve.

Example 7.4. Consider the $h$-vector

$$
(1,3,6,6,3,3,2) .
$$

The values in degrees 4 and 5 force the existence of a cubic curve of some sort in the base locus of $\left[I_{X}\right]_{4}$ and of $\left[I_{X}\right]_{5}$ for any finite set $X$ with this $h$-vector. We will look at a few different kinds of cubic curves to see how the differences in the geometry of the curves gives different behavior with respect to unexpected hypersurfaces (specifically cones). Our goal is not to give an exhaustive list of possible sets of points with this $h$-vector, but rather to highlight a few to see how they differ. So consider the following sets of points sharing this $h$-vector.

- Let $X$ consist of 18 points on a twisted cubic $C$ (note that the $h$-vector of these 18 points is $(1,3,3,3,3,3,2))$ plus 6 general points. Then we claim that $X$ admits no unexpected cone of any degree.

Notice that

$$
\operatorname{dim}\left[I_{X}\right]_{t}= \begin{cases}4 & \text { if } t=3 \\
16 & \text { if } t=4 \\
34 & \text { if } t=5 \\
\left(\begin{array}{c}
t+3 \\
3
\end{array}\right)-24 & \text { if } t \geq 6\end{cases}
$$

We first consider the case $t \leq 5$, and we notice edim $(X, t, t)=0$ in this case. Since any cone of degree $\leq 5$ containing $X$ must also contain $C$, it also contains the cone over $C$ (which is a surface of degree 3 ) as a component. But the projection from $P$ of the 6 general points gives 6 general points in the plane, and there is no conic through 6 general points. Thus $\operatorname{dim}\left[I_{X \cup P^{t}}\right]_{t}=0$ for $t \leq 5$ so $\operatorname{adim}(X, t, t)=\operatorname{edim}(X, t, t)=0$.

Now let $t \geq 6$. We have

$$
\operatorname{vdim}(X, t, t)=\left(\begin{array}{c}
t+3 \\
3
\end{array}\right)-24-\left(\begin{array}{c}
t+2 \\
3
\end{array}\right)=\left(\begin{array}{c}
t+2 \\
2
\end{array}\right)-24>0 .
$$

Now, the projection of the points on $C$ gives a set of points with $h$-vector $(1,2,3,3,3,3,3)$ so adding six general points gives a set with $h$-vector $(1,2,3,4,5,6,3)$, hence the general projection imposes independent conditions on plane curves of degree $t$. Thus the vector space dimension of this linear system (hence the vector space dimension of the family of cones of degree $t$ with vertex $P$ ) is the expected one.

Notice that if the $h$-vector had been $(1,3,6,6,3,3,3)$ then the projection would have $h$-vector $(1,2,3,3,3,3,3,1)$ and the above argument would not work for $t=6$. Indeed, Theorem 7.8 gives an unexpected sextic cone. 
- Let $C$ be a set of three disjoint lines. Let $X$ consist of 6 points on one of the lines and 7 points on each of the remaining lines, chosen generally, together with 4 general points in $\mathbb{P}^{3}$. (The $h$-vector of the points on $C$ is $(1,3,5,3,3,3,2)$, and the $h$-vector of $X$ is again $(1,3,6,6,3,3,2)$.) Then the expected dimension in degree 5 is 0 as before, but since there is a pencil of conics through four general points in the plane we obtain $\operatorname{adim}(X, 5,5)=2$, i.e. there is a pencil of unexpected cones of degree 5 .

- Let $C$ be a smooth plane cubic curve and let $X$ consist of 17 points on $C$ (with $h$-vector $(1,2,3,3,3,3,2))$ plus a set $X_{1}$ of 7 general points in $\mathbb{P}^{3}$. One can check that $1=A V_{X, 0}(5)=A V_{C \cup X_{1}, 0}(5)=A V_{X_{1}, 0}(2)$ in accordance with Corollary 6.5 but that there is no unexpected hypersurface because $\operatorname{adim}\left(X_{1}, 2,2\right)=0$.

- Let $C$ be a smooth plane cubic curve in $\mathbb{P}^{3}$, and let $\lambda_{1}$ and $\lambda_{2}$ be general lines in $\mathbb{P}^{3}$. Let $X$ consist of 17 general points on $C$, plus a subset $X_{1}$ of four general points on $\lambda_{1}$ and three general points on $\lambda_{2}$. One can check that $X$ also has the $h$-vector $(1,3,6,6,3,3,2)$ so we expect no surface of degree 5 with a point of multiplicity 5 at a general point $P$. However, the cone over $C \cup \lambda_{1} \cup \lambda_{2}$ is such a surface. But notice that the one-dimensional component of the base locus of $\left[I_{X}\right]_{5}$ is only the plane cubic.

Thus the $h$-vector $(1,3,6,6,3,3,2)$ may or may not force an unexpected cone, depending mostly, but not entirely, on the cubic curve that is forced by the $h$-vector.

It is worth noting that the $h$-vector $(1,3,6,5,3,3,2)$ (analyzed as above) admits an unexpected cone even when the cubic curve is a twisted cubic, and $(1,3,6,6,3,3,2)$ admits an unexpected cone even when the cubic is a plane cubic.

As mentioned at the beginning of this section, we do not believe that any finite $O$-sequence forces the existence of unexpected hypersurfaces for non-degenerate sets of points. However, some sequences force the existence of a curve in the base locus of at least some components of the ideal, and if this curve is not a plane curve then we can find finite $O$-sequences that force unexpected hypersurfaces. This idea is elementary, but a bit technical. Thus we will first look at an example, to make the proof of Theorem 7.8 clearer. We will refer to the notation of Theorem 7.8 in this example.

Example 7.5. Consider sets of points $X$ with the $h$-vector

$$
(1,3,6,9,8,7, \underbrace{6,6, \ldots, 6}_{\ell})
$$

where $\ell \geq 6$. In the notation of Theorem 7.8 we have $k=5, d=6, N=(6-4)+(9-$ $5)+(8-6)+(7-6)=9$ and $m=3$. Because of the values of this $h$-vector, Proposition 7.3 applies. We get that for $6 \leq t \leq \ell+5,\left(I_{X}\right)_{\leq t}$ has a 1-dimensional base locus, $C$, of degree 6 , together with a finite set $X_{2}$, which imposes independent conditions on hypersurfaces of degree $\geq 5$. In fact

$$
\left(I_{X}\right)_{\leq t}=\left(I_{C \cup X_{2}}\right)_{\leq t}
$$

for $6 \leq t \leq \ell+5$. Furthermore, using Lemma 7.7 as in the proof of Theorem 7.8, we see that $\left|X_{2}\right| \leq N=9$. Assume that $C$ is not a plane curve.

Now we look in degree 11 , which is in the range $6 \leq t \leq \ell+5$. Applying Theorem 6.4 we obtain

$$
A V_{X, 0}(11)=A V_{X_{2} \cup C, 0}(11)=A V_{X_{2}, 0}(5)+\left[\left(\begin{array}{c}
6-1 \\
2
\end{array}\right)-g\right]>0,
$$


where $g$ is the arithmetic genus of $C$. The fact that this is positive follows since $C$ is not a plane curve.

We remark that this works because the degree 11 is such that the value of the $h$-vector is still 6 in that degree. Beyond degree $k+\ell$ there is no longer a curve, and Theorem 6.4 no longer applies.

Now let $P$ be a general point in $\mathbb{P}^{3}$ and consider the projection from $P$ to a general $\mathbb{P}^{2}$. The image of $X_{2}$ is thus a set of $\leq 9$ points in the plane, and as such it lies on a plane curve of degree $m=3$ and hence also a plane curve of degree $k=5$. The cone over this curve is a surface of degree 5 containing $X_{2}$ with multiplicity 5 at $P$. Together with the cone over $C$ (which has degree 6 ), we have a surface of degree 11 having multiplicity 11 at $P$. This means $\operatorname{adim}(X, 11,11)>0$. Since also $A V_{X, 0}(11)>0, X$ admits an unexpected cone of degree 1 .

Lemma 7.6 ([BGM] Lemma 3.1). Let $I \subset R$ be an ideal satisfying $h_{R / I}(t)=\left(\begin{array}{c}t+m \\ t\end{array}\right)$ and $h_{R / I}(t+1)=\left(\begin{array}{c}t+1+m \\ t+1\end{array}\right)$. Then $[I]_{t}$ is the degree $t$ component of the saturated ideal of an $m$-dimensional linear space in $\mathbb{P}^{n}$ (and similarly for $[I]_{t+1}$ ).

Lemma 7.7. Let $C$ be a reduced, unmixed, non-degenerate curve in $\mathbb{P}^{3}$ of degree $d$. Let $h_{C}(t)$ be its Hilbert function. Then $\Delta h_{C}(t)$ has a sharp lower bound as follows: If $d=2$ or 3 then the lower bound is (respectively)

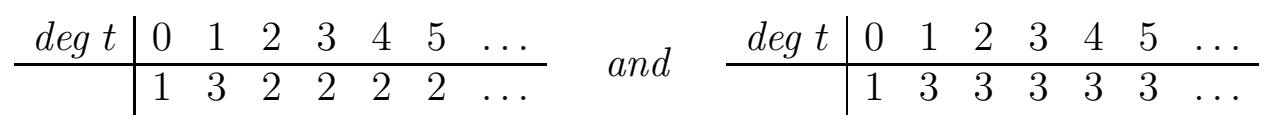

If $d \geq 4$ then the lower bound is

\begin{tabular}{c|cccccccccccc}
$\operatorname{deg} t$ & 0 & 1 & 2 & 3 & 4 & $\ldots$ & $d-3$ & $d-2$ & $d-1$ & $d$ & $d$ & $\ldots$ \\
\hline & 1 & 3 & 4 & 5 & 6 & $\ldots$ & $d-1$ & $d$ & $d$ & $d$ & $d$ & $\ldots$
\end{tabular}

Proof. In all cases, since $C$ is non-degenerate we must have $\Delta h_{C}(1)=3$. If $d=2$ and $C$ is non-degenerate then $C$ must be a pair of disjoint lines, and the first given $\Delta h_{C}$ is its Hilbert function. (So this is precisely the Hilbert function and not a lower bound.) If $d=3$ and $\Delta h_{C}(t) \leq 2$ for any $t \geq 2$ then by Macaulay it can never grow to 3 , which it must do since $\operatorname{deg} C=3$. Thus the first two cases are done.

Notice that the given sequence is $\Delta h_{C}$ for the curve $C$ consisting of the union of a plane curve of degree $d-1$ and a line, meeting at one point. Thus this sequence occurs.

Let $I_{C}$ be the saturated ideal of $C$. Since $R / I_{C}$ has depth $\geq 1$, if $L$ is a general linear form then the first difference of $h_{C}(t)$ is the Hilbert function of $R /\left(I_{C}, L\right)$ and so is an $O$-sequence. We know $\Delta h_{C}(t)=d$ for $t \gg 0$. If $\Delta h_{C}(2) \leq 2$ then by Macaulay's theorem it can never grow to $d$, so we must have $\Delta h_{C}(2) \geq 3$.

Suppose $\Delta h_{C}(2)=3$. In order to eventually reach $d$, by Macaulay's theorem we must have $\Delta h_{C}(t)=t+1$ for $2 \leq t \leq d-1$. Then by Lemma 7.6 (taking $\left.m=1\right),\left[\left(I_{C}+(L)\right) /(L)\right]_{t}$ is the degree $t$ component of a line in $K[x, y, z]$. Thus since $I_{C}$ is saturated, $\left[I_{C}\right]_{t}$ is the degree $t$ component of a plane, i.e. $C$ is a plane curve of degree $d$. This is impossible since $C$ is non-degenerate.

The same argument applies for all degrees $3 \leq t \leq d-2$ : we must have $\Delta h_{C}(t) \geq t+1$ in order to reach $d$, and if we have equality then $C$ must be a plane curve. Thus the stated Hilbert function is the smallest possible.

If Conjecture 7.1 is true, the following kind of result is the best that one can hope for, in terms of finding a Hilbert function that forces unexpectedness (but we do not claim that 
this result is optimal in any way). It says that for a certain class of Hilbert functions (which we define specifically via some numerical conditions) for which a curve is forced in some component of the ideal because of maximal growth, if you assume that this curve is not a plane curve, then any set of points with this Hilbert function must admit unexpected cones. Conjecture 7.1 thus implies that if, however, you allow the curve to be a plane curve then a set of points can be found for which there is no unexpected cone.

Theorem 7.8. Let $X$ be a set of points in $\mathbb{P}^{3}$ with h-vector

$$
(1,3, a_{2}, a_{3}, \ldots, a_{k}, \underbrace{d, d, \ldots, d}_{\ell})
$$

where $k \geq 2$ and $a_{k}>d$. Assume

$$
2 \leq d \leq \min \{k+1, \ell\}
$$

In case $d \geq 4$, let

$$
b_{i}= \begin{cases}a_{i}-(i+2) & \text { for } 2 \leq i \leq d-2 \\ a_{i}-d & \text { for } d-1 \leq i \leq k .\end{cases}
$$

If $d=2$ or $d=3$, we replace $i+2$ by the bounds given in the first two parts of Lemma 7.7.

Set

$$
N=\sum_{i=2}^{k} b_{i}
$$

and

$$
m=\min \left\{i \mid\left(\begin{array}{c}
i+2 \\
2
\end{array}\right)>N\right\} \text {. }
$$

We also assume $m \leq k$.

Let $C$ be the equidimensional curve of degree d guaranteed by Proposition 7.3. If $C$ is not a plane curve then $X$ admits an unexpected cone of degree $d+k$.

Proof. Since $d \leq k+1$, Proposition 7.3 applies in degree $k$. In particular, we get from Proposition 7.3 (c) that $X_{2}$ imposes independent conditions on $\left[I_{X}\right]_{s}$ for any $s \geq k$, hence it also imposes independent conditions on the complete linear system of forms of degree $s$; we will use the case $s=k$.

Now we look in degree $d+k$. By Theorem 6.4 we have

$$
A V_{C \cup X_{2}}(d+k)=A V_{X_{2}, 0}(k)+\left[\left(\begin{array}{c}
d-1 \\
2
\end{array}\right)-g\right]>0
$$

since $C$ is not a plane curve.

Note that $N$ is an upper bound for $\left|X_{2}\right|$, thanks to Lemma 7.7. If we denote by $\pi_{P}$ the projection from a general point $P$ to a general plane, the assumption $m \leq k$ guarantees that $\pi_{P}\left(X_{2}\right)$ lies on a curve of degree $k$. This means that $X_{2}$ lies on a cone of degree $k$ with vertex at $P$. If $S_{P}$ is the cone over $C$ with vertex $P$, the union of these cones is a surface of degree $d+k$ with multiplicity $d+k$ at $P$. Thus $\operatorname{adim}\left(C \cup X_{2}, d+k, d+k\right)>0$, so we have an unexpected cone of degree $d+k$ for $C \cup X_{2}$. But $k+1 \leq d+k \leq k+\ell$ so $\left[I_{X}\right]_{d+k}=\left[I_{C \cup X_{2}}\right]_{d+k}$, so also $X$ admits an unexpected cone of degree $d+k$.

Example 7.9. As mentioned above, the preceding result is not meant to be optimal. Consider for instance the $h$-vector $(1,3,6,5,3,3,3)$. We have $d=3, \ell=3, N=3+2=5$, $m=2, k=3$. The cubic curve $C$ guaranteed in the base locus of $\left[I_{X}\right]_{t}$ for $t=4,5,6$ is either 
a twisted cubic, the union of a line and a conic (meeting in 0 or 1 points), or the union of three lines (meeting in a total of $<3$ points). Considering possible Hilbert functions of such curves, the given $h$-vector forces a set $X_{2}$ of at most $N=5$ points off the curve (as a result of Lemma 7.7).

The theorem guarantees an unexpected cone of degree 6. Indeed,

$$
\operatorname{dim}\left[I_{X}\right]_{6}-\left(\begin{array}{c}
6-1+3 \\
3
\end{array}\right)=60-56=4
$$

and since the projection of $\leq 5$ points to $\mathbb{P}^{2}$ lies on at least a 5 -dimensional vector space of plane cubics, the cones over these cubics (with vertex at the general point $P$ ) together with the cone over $C$ confirm the conclusion that there is an unexpected sextic.

However, these projected points also lie on at least one conic, so there is a quadric cone containing $X_{2}$, and together with the cone over $C$ we get an unexpected quintic cone (since $\left.\operatorname{dim}\left[I_{X}\right]_{5}-\left(\begin{array}{c}5-1+3 \\ 3\end{array}\right)=0\right)$, which is not covered by the theorem.

If we had allowed $C$ to be a plane cubic curve, Lemma 7.7 would no longer hold: the lower bound in this case would be given by the sequence $(1,2,3,3,3, \ldots)$ so $\left|X_{2}\right|$ could also be 6 .

The following result gives a geometric property for a set of points that is enough to find $h$-vectors that force unexpected cones.

Corollary 7.10. Let $X$ be a set of points in $\mathbb{P}^{3}$ in linear general position, and assume that $X$ has h-vector given by the numerical conditions in Theorem 7.8 . Then $X$ admits an unexpected cone.

Proof. The assumption of linear general position forces $C$ to be non-degenerate.

\section{REFERENCES}

[ABR] J. Abbott, A.M. Bigatti, and L. Robbiano, CoCoA : a system for doing Computations in Commutative Algebra, available at http://cocoa.dima.unige.it.

[A] S. Akesseh, Ideal Containments under Flat Extensions and Interpolation on Linear Systems in $\mathbb{P}^{2}, \mathrm{PhD}$ thesis, University of Nebraska, 2017.

[AL] D. Alberelli and P. Lella, Strongly stable ideals and Hilbert polynomials, J. Software for Algebra and Geom. 9 (2019) 1-9.

[AH] J. Alexander and A. Hirschowitz, An asymptotic vanishing theorem for generic unions of multiple points, Invent. Math. 140:2 (2000) 303-325.

[BDSSS] T. Bauer, S. Di Rocco, D. Schmitz, T. Szemberg and J. Szpond, On the postulation of lines and a fat line, J. Symb. Comp., 91 (2019) 3-16.

[BGM] A. Bigatti, A.V. Geramita and J. Migliore, Geometric consequences of extremal behavior in a theorem of Macaulay, Trans. Amer. Math. Soc. 346, no. 1 (1994), 203-235.

$[\mathrm{BH}] \quad$ W. Bruns and J. Herzog, "Cohen-Macaulay rings," Cambridge Studies in Advanced Mathematics, 39. Cambridge University Press, Cambridge, 1993.

[CCG] E. Carlini, M.V. Catalisano and A.V. Geramita. On the Hilbert function of lines union one nonreduced point. Annali della Scuola Normale Superiore di Pisa. Classe di scienze. 2016;15(1):69-84.

[CaS] G. Caviglia and E. Sbarra, Zero-generic initial ideals, Manus. Math. 148 (2015) 507-520.

[CM] L. Chiantini and J. Migliore, Sets of points which project to complete intersections, preprint 2019 (arXiv:1904.02047).

[CoS] A. Conca and J. Sidman, Generic initial ideals of points and curves, J. Symb. Comp. 40(3) (2005) 1023-38.

[CHMN] D. Cook, B. Harbourne, J. Migliore and U. Nagel, Line arrangements and configurations of points with an unexpected geometric property, Compositio Math. 154:10 (2018) 2150-2194. 
[CH] S. Cooper and B. Harbourne, Regina Lectures on Fat Points, pp. 147-187, in: Connections Between Algebra, Combinatorics, and Geometry, S. Cooper and S. Sather-Wagstaff editors, vol. 76 of Springer proceedings in mathematics \& statistics, 2014.

[DIV] R. Di Gennaro, G. Ilardi and J. Vallès, Singular hypersurfaces characterizing the Lefschetz properties, J. London Math. Soc. (2) 89 (2014), no. 1, 194-212.

[DMO] M. Di Marca, G. Malara, A. Oneto, Unexpected curves arising from special line arrangements, to appear in J. Alg. Comb. (arXiv:1804.02730).

[DHRST] M. Dumnicki, B. Harbourne, J. Roé, T. Szemberg and H. Tutaj-Gasińska, Unexpected surfaces singular on lines in $\mathbb{P}^{3}$, arXiv:1901.03725.

[E] D. Eisenbud, An Introduction to Commutative Algebra with a View Towards Algebraic Geometry, 1995 Springer-Verlag, New York.

[FGST] E. Farnik, F. Galuppi, L. Sodomaco and W. Trok, On the unique unexpected quartic in $\mathbb{P}^{2}$, J. Algebr. Comb. (2019) on-line (arXiv:1804.03590).

[Gi] A. Gimigliano, On linear systems of plane curves, Thesis, Queen's University, Kingston, 1987.

[Gr] M. Green, Generic initial ideals, In: Six Lectures on Commutative Algebra. Bellaterra, 1996. In: Progr. Math., vol. 166. Birkhäuser, Basel, pp. 119-186.

[GLP] L. Gruson, R. Lazarsfeld and C. Peskine, On a theorem of Castelnuovo, and the equations defining space curves, Invent. Math. 72 (1983), no. 3, 491-506.

[GS] D.R. Grayson and M.E. Stillman, Macaulay 2, a software system for research in algebraic geometry, (2002).

$[\mathrm{H}] \quad$ B. Harbourne, The geometry of rational surfaces and Hilbert functions of points in the plane, Proceedings of the 1984 Vancouver Conference in Algebraic Geometry, CMS Conf. Proc. 6, Amer. Math. Soc., Providence, RI (1986), 95-111.

$[\mathrm{HaH}] \quad$ K. Hanumanthu and B. Harbourne, Real and complex supersolvable line arrangements in the projective plane, arXiv:1907.07712.

[HMNT] B. Harbourne, J. Migliore, U. Nagel and Z. Teitler, Unexpected hypersurfaces and where to find them, to appear in Michigan Math. J. (arXiv: 1805.10626).

[HMT] B. Harbourne, J. Migliore and H. Tutaj-Gasińska, New constructions of unexpected hypersurfaces in $\mathbb{P}^{n}$, Rev. Mat. Compl. (2020) on-line (arXiv:1904.03251).

[Hrm] T. Harima, Characterization of Hilbert functions of Gorenstein Artin algebras with the Weak Stanley Property, Proc. Amer. Math. Soc. 123 (1995), 3631-3638.

[Hart] R. Hartshorne, Connectedness of the Hilbert scheme, Math. Inst. des Hautes Etudes Sci, 29 (1966) 261-304.

[HaHi] R. Hartshorne and A. Hirschowitz, Droites en position générale dans l'espace projectif, Algebraic geometry (La Rábida, 1981), 169-188, Lecture Notes in Math., 961, Springer-Verlag 1982.

[Hi] A. Hirschowitz, Une conjecture pour la cohomologie des diviseurs sur les surfaces rationelles génériques, J. Reine Angew. Math. 397 (1989), 208-213.

[Hu] H. A. Hulett, Maximum betti numbers of homogeneous ideals with a given hilbert function, Comm. Algebra, 21:7 (1993) 2335-2350.

[LU] A. Laface and L. Ugaglia, A conjecture on special linear systems of $\mathbb{P}^{3}$, Rend. Sem. Mat. Univ. Pol. Torino - Vol. 63, 1 (2005).

[Mac] F.S. Macaulay, Some properties of enumeration in the theory of modular systems, Proc. London Math. Soc. 26 (1927), 531-555.

[Mi] J. Migliore, "Introduction to liaison theory and deficiency modules," Birkhäuser, Progress in Mathematics vol. 165 (1998).

[MN] J. Migliore and U. Nagel, Lifting monomial ideals, Comm. Algebra 28:12 (2000) 5679-5701.

[Se] B. Segre, Alcune questioni su insiemi finiti di punti in geometria algebrica, Atti del Convegno Internazionale di Geometria Algebrica (Torino, 1961), Rattero, Turin, 1962, 15-33.

[S1] J. Szpond, Unexpected curves and Togliatti-type surfaces, Math. Nach. 293 (2020) 158-168 (arXiv:1810.06607).

[S2] J. Szpond, Unexpected hypersurfaces with multiple fat points, arXiv:1812.04032.

[Tr] W. Trok, Projective Duality, Unexpected Hypersurfaces and Logarithmic Derivations of Hyperplane Arrangements, in preparation (2019).

[V] G. Valla, On the Betti numbers of perfect ideals, Comp. Math. 91:3 (1994) 305-319. 
Dipartimento di Matematica e Informatica, Università di Catania, Viale A. Doria, 6, I95125 CATANia, Italy

E-mail address: favacchio@dmi.unict.it

Dipartimento di Matematica e Informatica, Università di Catania, Viale A. Doria, 6, I95125 Catania, Italy

E-mail address: guardo@dmi.unict.it

Department of Mathematics, University of Nebraska, Lincoln, NE 68588-0130 USA

E-mail address: bharbourne1@unl.edu

Department of Mathematics, University of Notre Dame, Notre Dame, IN 46556 USA

E-mail address: migliore.1@nd.edu 